\title{
The arithmetic of Prym varieties in genus 3
}

\author{
Nils Bruin
}

\begin{abstract}
Given a curve of genus 3 with an unramified double cover, we give an explicit description of the associated Prym variety. We also describe how an unramified double cover of a nonhyperelliptic genus 3 curve can be mapped into the Jacobian of a curve of genus 2 over its field of definition and how this can be used to perform Chabauty- and Brauer-Manin-type calculations for curves of genus 5 with an fixed-point-free involution. As an application, we determine the rational points on a smooth plane quartic and give examples of curves of genus 3 and 5 violating the Hasse principle. The methods are, in principle, applicable to any genus 3 curve with a double cover. We also show how these constructions can be used to design smooth plane quartics with specific arithmetic properties. As an example, we give a smooth plane quartic with all 28 bitangents defined over $\mathbb{Q}(t)$. By specialization, this also gives examples over $\mathbb{Q}$.
\end{abstract}

\section{Introduction}

In this article, we investigate the arithmetic of unramified double covers of non-hyperelliptic curves of genus 3. This research is inspired by the recent success in applying the theory of unramified double covers of hyperelliptic curves to the problem of determining the set of rational points on such curves [Bru02, Bru03, BF05, Wet97]. Combined with explicit Chabauty methods, this has yielded very practical methods for obtaining often sharp bounds on the number of rational points on a hyperelliptic curve. In the hyperelliptic case, the construction of unramified covers is particularly easy to make explicit using Kummer theory. There has been some limited work investigating how these ideas may be generalized to unramified covers of higher degree of hyperelliptic curves [BF03].

In this article, we generalize in a different direction. We derive the general form of a curve $C$ of genus 3 over a field $K$ of characteristic zero that allows an unramified double cover $\pi: D \rightarrow C$ defined over $K$. A non-hyperelliptic curve of genus 3 with an unramified double cover allows a smooth plane projective model of the form

$$
C: Q_{1}(u, v, w) Q_{3}(u, v, w)=Q_{2}(u, v, w)^{2},
$$

where $Q_{1}, Q_{2}, Q_{3} \in K[u, v, w]$ are quadratic forms, such that the unramified double cover of $C$ has a canonical model in $\mathbb{P}^{4}$ of the form

$$
D:\left\{\begin{array}{l}
Q_{1}(u, v, w)=r^{2} \\
Q_{2}(u, v, w)=r s \\
Q_{3}(u, v, w)=s^{2}
\end{array}\right.
$$

Received 2 June 2006, accepted in final form 16 July 2007, published online 14 March 2008.

2000 Mathematics Subject Classification 11G30 (primary), 14H40 (secondary).

Keywords: Prym varieties, Chabauty methods, rational points on curves, covering techniques, Brauer-Manin, smooth plane quartics.

The research in this paper is partially supported by NSERC.

This journal is (C) Foundation Compositio Mathematica 2008. 


\section{N. BRUIN}

We also describe how the associated Prym variety (the connected component of the kernel of $\pi_{*}: \operatorname{Jac}(D) \rightarrow \operatorname{Jac}(C)$ that contains the origin $O$ of $\left.\operatorname{Jac}(D)\right)$ can be described explicitly as $\operatorname{Jac}(F)$ of some genus 2 curve $F$ over $K$ :

$$
F: y^{2}=-\operatorname{det}\left(M_{1}+2 x M_{2}+x^{2} M_{3}\right),
$$

where $M_{i}$ is the symmetric $3 \times 3$ matrix such that

$$
(u, v, w) M_{i}\left(\begin{array}{c}
u \\
v \\
w
\end{array}\right)=Q_{i}(u, v, w) .
$$

Combined with earlier work, this gives a complete description of how principally polarized Abelian surfaces arise as Prym varieties in genus 3 over base fields of characteristic zero which are not necessarily algebraically closed (see Theorem 5.1).

The description of $\operatorname{Prym}(D / C)$ as $\operatorname{Jac}(F)$ over $\mathbb{C}$ can already be found in [ACGH85, Exercise VI.F]. For arithmetic applications, we need the result in a more general setting. If $C$ has a rational point, then the trigonal construction gives a Galois-theoretic way of describing the Prym variety as the Jacobian of some curve (see [Don92] and [Rec74]). Here, we refine the description to arbitrary characteristic zero base fields. The results should generalize to base fields of arbitrary odd characteristic.

We show how $D$ can be mapped into $\operatorname{Prym}(D / C)$ over $K$, without an explicit rational degree one divisor class on $D$ (see Proposition 6.2). We can thus use Chabauty-like methods on genus 5 curves in Abelian surfaces to obtain what is, in practice, often a sharp bound on the number of rational points on $D$. This can, in turn, be used to obtain a corresponding bound on the number of rational points on $C$. As an example, we prove the following.

Proposition 1.1. For the curve

$$
C:\left(4 u^{2}-4 v w+4 w^{2}\right)\left(2 u^{2}+4 u v+4 v^{2}\right)=\left(2 u^{2}+2 u w-4 v w+2 w^{2}\right)^{2}
$$

we have $C(\mathbb{Q})=\{(0: 1: 0)\}$.

The proof avoids computing the Mordell-Weil group of $\operatorname{Jac}(C)$. Instead, it uses the computationally more accessible Mordell-Weil group of the associated Prym variety, which is an Abelian surface.

Remark 1.2. The method does not put restrictions on the geometry of $C$. Indeed, let $L$ be a field extension of $K$ such that the group scheme $\operatorname{Jac}(C)[2]$ has a non-trivial rational point. Let $d=[L: K]$. We can use the construction above to obtain an unramified double cover $D_{L} / C_{L}$ and a corresponding map $D_{L} \rightarrow \operatorname{Prym}\left(D_{L} / C_{L}\right)$. In complete analogy to the treatment of genus 2 in [Bru03], we take Weil restrictions and obtain a finite étale cover of dimension $d$ varieties $\Re_{L / K}\left(D_{L}\right) \rightarrow \Re_{L / K}\left(C_{L}\right)$ and a map $\Re_{L / K}\left(D_{L}\right) \rightarrow \Re_{L / K}\left(\operatorname{Prym}\left(D_{L} / C_{L}\right)\right)$. Inside $\Re_{L / K}\left(C_{L}\right)$ we have $C=C_{K}$ and we can take its preimage $\tilde{D} \subset \Re_{L / K}\left(D_{L}\right)$. This will give a degree $2^{d}$ unramified cover of $C$, which could consist of multiple components, depending on the linear dependencies between the $L / K$-conjugates of the 2-torsion point in $\operatorname{Jac}(C)[2]$ corresponding to $D_{L} / C_{L}$.
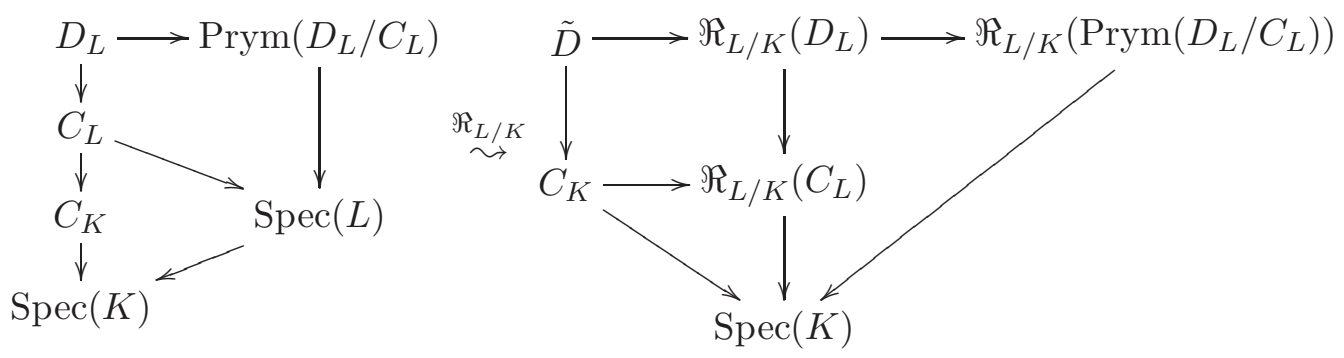


\section{The ARIThmetic of Prym VARIETIES IN Genus 3}

In any case, we end up with an unramified cover $\tilde{D} / C$ over $K$ that maps non-trivially into an Abelian variety of dimension $2 d$, which has a Mordell-Weil group that is computationally relatively accessible, because it is isomorphic to $\operatorname{Prym}\left(D_{L} / C_{L}\right)(L)$. We can then apply Chabauty-like methods to determine $\tilde{D}(K)$ and $C(K)$. The group $\Re_{L / K}\left(\operatorname{Prym}\left(D / C_{L}\right)\right)(K) \simeq \operatorname{Prym}\left(D / C_{L}\right)(L)$ would be the hardest ingredient to obtain and it should be noted that the computations involved would probably be prohibitive, except for very low degree $L$.

Since the mapping of $D$ into $\operatorname{Prym}(D / C)$ does not require a rational point on $D$, we can apply this construction to prove in examples that $D(\mathbb{Q})$ is empty, even if $D$ does have points everywhere locally. In our computations, we show in certain examples that the image of $D\left(\mathbb{A}_{\mathbb{Q}}\right)$ in $\operatorname{Prym}(D / C)\left(\mathbb{A}_{\mathbb{Q}}\right)$ misses the closure of $\operatorname{Prym}(D / C)(\mathbb{Q})$ by combining the local information at a finite number of primes.

It is reassuring that, at least conjecturally, our computations correspond to determining part of the Brauer-Manin obstruction of $D$. By this we mean that if the argument above succeeds in showing that $D$ has no rational points, then subject to standard conjectures, there is a BrauerManin obstruction to having rational points, i.e. $D\left(\mathbb{A}_{\mathbb{Q}}\right)^{\mathrm{Br}}$ is empty. The explanation below is of no relevance for the rest of this paper, but may be of interest to experts.

Suppose that $D$ is a curve of genus at least 2, defined over a number field $K$, with points everywhere locally. Since $D$ is naturally a subvariety of $\underline{\operatorname{Pic}^{1}}(D)$, the scheme representing the functor $X \mapsto \operatorname{Pic}^{1}(D / X)$, it follows that $\underline{\operatorname{Pic}^{1}}(D)$ has points everywhere locally as well. Since $\operatorname{Pic}^{1}(D)$ is a torsor under $\operatorname{Jac}(D)$, it follows that $\underline{\mathrm{Pic}^{1}}$ represents an element of $\amalg(\operatorname{Jac}(D) / K) \subset H^{1}(K, \operatorname{Jac}(D))$. Manin has proved (see [Sko01, Theorem 6.2.3]) that if $\amalg(\operatorname{Jac}(D) / K$ ) is finite, then the failure of any $X \in \amalg(\operatorname{Jac}(D) / K)$ to have points globally, can be explained by the Brauer-Manin obstruction.

If $\underline{\operatorname{Pic}^{1}}(D)$ does have a rational point then it is isomorphic to $\operatorname{Jac}(D)$ and hence $D$ can be considered as a subvariety of $\operatorname{Jac}(D)$ (via the Abel-Jacobi map). In this case, Scharaschkin [Sch99] proves that if $\amalg(\operatorname{Jac}(D) / K)$ has trivial divisible subgroup, then $D\left(\mathbb{A}_{K}\right)_{\bullet}^{\mathrm{Br}}$ is a subset of $D\left(\mathbb{A}_{K}\right) \bullet \cap$ $\overline{\operatorname{Jac}(D)(K)}$. Here, the $\bullet$ symbol means taking the quotient by the connected component. This only makes a difference at the archimedean components of adelic point sets.

If $D$ does have a degree one divisor, then our map $2\left(\iota_{*}-\mathrm{id}_{*}\right): D \rightarrow \operatorname{Prym}(D / C)$ differs by a translation over a rational point of $\operatorname{Prym}(D / C)$ from twice the map obtained via the obvious projection $J(D) \rightarrow \operatorname{Prym}(D / C)$. Hence, if $2\left(\iota_{*}-\mathrm{id}_{*}\right) D\left(\mathbb{A}_{K}\right) \bullet \cap \overline{\operatorname{Prym}(D / C)(\mathbb{Q})}$ • is empty, then so is $D\left(\mathbb{A}_{K}\right) \bullet \cap \overline{\operatorname{Jac}(D)(K)}$. and, therefore, $D$ conjecturally has a non-trivial Brauer-Manin obstruction to having rational points.

Proposition 1.3. The genus 5 curve

$$
D:\left\{\begin{array}{l}
\left(v^{2}+v w-w^{2}\right)=r^{2} \\
\left(u^{2}-v^{2}-w^{2}\right)=r s \\
\left(u v+w^{2}\right)=s^{2}
\end{array}\right.
$$

and the genus 3 curve

$$
C:\left(v^{2}+v w-w^{2}\right)\left(u v+w^{2}\right)=\left(u^{2}-v^{2}-w^{2}\right)^{2}
$$

over $\mathbb{Q}$ both have points everywhere locally, but they have no rational points.

For this example, we find $\operatorname{Prym}(D / C)(\mathbb{Q}) \simeq \mathbb{Z} \times \mathbb{Z}$. This illustrates that the method used is not a special case of a Chabauty-type argument.

Also note that while, at least conjecturally, the curve $D$ in Proposition 1.3 has a non-trivial Brauer-Manin obstruction to having points, the same does not immediately follow for $C$. Hence, the proof of Proposition 1.3 does not suggest that $C\left(\mathbb{A}_{\mathbb{Q}}\right)^{\mathrm{Br}}$ is empty, although undoubtedly it is. 


\section{N. BRUIN}

In addition, we investigate the arithmetic implications of the geometric description of the fibres of the Prym map between moduli spaces, corresponding to the functor Prym : $\{\pi: D \rightarrow C\} \mapsto$ $\{\operatorname{Prym}(D / C)\}$, given in [Ver87]. For a general principally polarized Abelian surface $A$ we denote the standard map $\mathbb{Z} \rightarrow \operatorname{End}(A)$ by $n \mapsto[n]$. Kummer surface $\mathcal{K}=A /\langle[-1]\rangle$ has a singular quartic model in $\mathbb{P}^{3}$, with a singular locus consisting of 16 points, corresponding to $A[2]$. Translation by $A[2]$ induces automorphisms on $\mathcal{K}$, given by linear transformations on $\mathbb{P}^{3}$. We write $\widehat{\mathbb{P}}^{3}$ for the space of planes in $\mathbb{P}^{3}$. Verra [Ver87] shows that over $\mathbb{C}$, the fibre of Prym over a principally polarized surface $A$ is birational to $\widehat{\mathbb{P}}^{3} / A[2]$. In fact, he gives a very precise description of the fibre as a blow-up of this space, where the exceptional components contain the moduli points corresponding to hyperelliptic or degenerate curves $C$.

In particular, a non-hyperelliptic genus 3 curve $C$ over $\mathbb{C}$ which has a double cover $D$ such that $\operatorname{Prym}(D / C)=A$ can be obtained as a plane section of $\mathcal{K}$, with $D$ the pull-back of $C$ to $A$. Any two such plane sections of $\mathcal{K}$ in the same $A[2]$-orbit give isomorphic covers $D / C$.

In this article we explain how, given a genus 2 curve $F$ over a number field $K$ and a genus 3 plane section $C$ of $\mathcal{K}=\operatorname{Jac}(F) /\langle \pm 1\rangle$, we can obtain a model for $C$ of the type $Q_{1} Q_{3}=\left(Q_{2}\right)^{2}$. Since a sufficiently general plane section of $\mathcal{K}$ is non-singular and thus of genus 3 , it shows that any Jacobian of a genus 2 curve over $K$ can be realized as a Prym variety of a non-hyperelliptic genus 3 curve over $K$. In fact, this particular construction certainly works over any odd characteristic base field with sufficiently many elements. We can phrase this in entirely elementary terms as follows.

Proposition 1.4. Let $k$ be an infinite field of odd characteristic and let $f \in k[x]$ be a square-free polynomial of degree 5 or 6 . Then there exist symmetric matrices $M_{1}, M_{2}, M_{3} \in \mathbb{Q}^{3 \times 3}$ such that

$$
f=\operatorname{det}\left(M_{1}+x M_{2}+x^{2} M_{3}\right) .
$$

We use this construction to obtain a systematic way of constructing curves of genus 3 with all 28 bitangents defined over a non-algebraically closed field, for instance $\mathbb{Q}(t)$ (see $\S 7$ ). By specialization of $t$, this gives infinitely many examples of curves over $\mathbb{Q}$ with rational bitangents. This strengthens a result in [Edg94], where an example is given with all bitangents defined over $\mathbb{R}$. See [Sch98] or [Cay79] for an approach via interpolation.

Finally, it should be noted that not all covers $\pi: D \rightarrow C$ defined over $K$ with $\operatorname{Prym}(D / C)=A$ and $C$ non-hyperelliptic, have $C$ occurring as a plane section of the associated Kummer surface $\mathcal{K}$ defined over $K$, since not all rational points of $\widehat{\mathbb{P}}^{3} / A[2]$ are covered by rational points of $\widehat{\mathbb{P}}^{3}$. This follows, for instance, from the fact that this does not even hold for the quotient by one 2-torsion point, i.e.

$$
\mathbb{P}^{3} /\left\langle\left(\begin{array}{rrrr}
1 & 0 & 0 & 0 \\
0 & 1 & 0 & 0 \\
0 & 0 & -1 & 0 \\
0 & 0 & 0 & -1
\end{array}\right)\right\rangle
$$

has rational points that do not lift to $\mathbb{P}^{3}$.

\section{Preliminaries}

First we fix some notation. Let $K$ be a field and let $C$ be a complete, absolutely irreducible algebraic curve over $K$. We write $\kappa_{C}$ for a canonical divisor on $C$ over $K$. For a divisor $\mathfrak{D} \in \operatorname{Div}(C)$, we write $[\mathfrak{D}]$ for its class in $\operatorname{Pic}(C)$ and $|\mathfrak{D}|$ for the complete linear system corresponding to $\mathfrak{D}$ and $l(\mathfrak{D})=\operatorname{dim}|\mathfrak{D}|$. We say that a divisor $\mathfrak{D}$ on $C$ is a $g_{d}^{r}$ if $\operatorname{deg}(\mathfrak{D})=d$ and $l(\mathfrak{D})>r$. We write $W_{d}^{r} \subset \operatorname{Pic}^{d}(C)$ for the classes of all such $g_{d}^{r}$. By abuse of notation, we also write $W_{d}^{r}$ for the corresponding subscheme of the scheme representing the functor $\operatorname{Pic}^{d}(C)$. 


\subsection{Unramified double covers and Prym varieties}

Let $\pi: D \rightarrow C$ be an unramified finite morphism of degree two between curves over a field $K$ and let $\iota: D \rightarrow D$ be the non-trivial involution of $D$ over $C$. It follows by the Riemann-Hurwitz theorem that $g(C)>0$ and that

$$
g(D)=2 g(C)-1 .
$$

The kernel of $\pi_{*}: \operatorname{Jac}(D) \rightarrow \operatorname{Jac}(C)$ has two connected components. The component that contains 0 coincides with the image of $\left(\mathrm{id}_{*}-\iota_{*}\right): \operatorname{Jac}(D) \rightarrow \operatorname{Jac}(D)$.

Definition 2.1. Let $\pi: D \rightarrow C$ be an unramified finite morphism of degree two between curves over a field $K$. We write $\operatorname{Prym}(\pi)=\operatorname{Prym}(D / C)$ for the connected component of the identity-element of $\operatorname{ker}\left(\pi_{*}: \operatorname{Jac}(D) \rightarrow \operatorname{Jac}(C)\right)$. We call this the Prym variety of $D / C$.

Thus, $\operatorname{Prym}(D / C)$ is an Abelian subvariety of $\operatorname{Jac}(D)$. The principal polarization on $\operatorname{Jac}(D)$ restricts to a principal polarization on $\operatorname{Prym}(D / C)$. Historically, Prym varieties were considered interesting primarily because they give examples of principally polarized Abelian varieties that are not Jacobian varieties. However, if $\operatorname{dim}(\operatorname{Prym}(D / C)) \leqslant 2$, then $\operatorname{Prym}(D / C)$ generally is a Jacobian variety. ${ }^{1}$ See $[\mathrm{ACGH} 85, \mathrm{VI}-\mathrm{C}]$ or $[\mathrm{Mum} 74]$ for details.

\subsection{Prym varieties in the hyperelliptic case}

In contrast to the general situation, the Prym variety associated to an unramified double cover of a hyperelliptic curve is closely related to a Jacobian variety. In fact, the Prym variety is isomorphic to the product of Jacobian varieties of subcovers, which themselves are again hyperelliptic.

Let us first assume that $C$ is a double cover of a $\mathbb{P}^{1}$ defined over a field $K$ of odd characteristic. Then $C$ has an affine model of the form

$$
C: y^{2}=f(x)
$$

where $f \in K[x]$ is a square-free polynomial of degree $2 g(C)+2$. Kummer theory tells us exactly what the unramified degree two extensions of $K(C)$ are. For any factorization $f=f_{1} f_{2}$, with $f_{1}, f_{2} \in K[x]$ and of even degree, we have a curve $D$ given by the affine model

$$
\left\{\begin{array}{l}
y_{1}^{2}=f_{1}(x) \\
y_{2}^{2}=f_{2}(x)
\end{array}\right.
$$

and an unramified morphism of degree two:

$$
\begin{aligned}
& \pi: D \quad D \quad C \\
& \left(x, y_{1}, y_{2}\right) \mapsto\left(x, y_{1} y_{2}\right)=(x, y) .
\end{aligned}
$$

Then there are the two obvious curves

$$
\begin{aligned}
& F_{1}: y_{1}^{2}=f_{1}(x) \\
& F_{2}: y_{2}^{2}=f_{2}(x)
\end{aligned}
$$

\footnotetext{
${ }^{1}$ It can also be the product of two elliptic curves, in which case it is a Jacobian of a reducible curve.
} 


\section{N. BRUIN}

with the obvious projections $\pi_{1}: D \rightarrow F_{1}$ and $\pi_{2}: D \rightarrow F_{2}$. This yields the familiar diagram associated to biquadratic extensions.

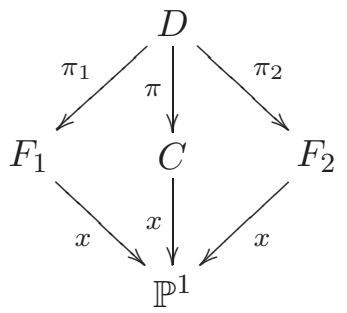

In addition, if $f_{1}, f_{2}$ are quadratic conjugate over some quadratic extension $L$ over $K$ then this construction will also yield an unramified double cover $D$ over $C$, defined over $K$, because the construction of $D$ is symmetric in $F_{1}, F_{2}$.

Proposition 2.2. Let $C, D, F_{1}, F_{2}, \pi, \pi_{1}, \pi_{2}$ be defined as above. Then

$$
\pi_{1}^{*}+\pi_{2}^{*}: \operatorname{Jac}\left(F_{1}\right) \times \operatorname{Jac}\left(F_{2}\right) \rightarrow \operatorname{Prym}(D / C)
$$

is an isomorphism of Abelian varieties.

Proof. First, we prove that $\pi_{1}^{*}$ indeed maps $\operatorname{Jac}\left(F_{1}\right)$ into $\operatorname{Prym}(D / C)$. To that end, take the generic point $\left(x_{1}, y_{1}\right) \in F_{1}$. We have $\pi_{1}^{*}\left(x_{1}, y_{1}\right)=\left(x_{1}, y_{1}, \sqrt{f_{2}\left(x_{1}\right)}\right)+\left(x_{1}, y_{1},-\sqrt{f_{2}\left(x_{1}\right)}\right)$. Under $\pi$, this maps to the divisor cut out by $x=x_{1}$. This shows that $\pi_{*} \pi_{1}^{*}: \operatorname{Jac}\left(F_{1}\right) \rightarrow \operatorname{Jac}(C)$ is constant and, hence, $\left.\left(\pi_{*} \pi_{1}^{*}\right)\right|_{\operatorname{Jac}\left(F_{1}\right)}$ is the zero map. By symmetry it follows that $\operatorname{Jac}\left(F_{1}\right) \times \operatorname{Jac}\left(F_{2}\right)$ lands in the kernel of $\pi_{*}$ under $\pi_{1}^{*}+\pi_{2}^{*}$ and, since it is connected and covers $0 \in \operatorname{Jac}(D)$, lands in $\operatorname{Prym}(D / C)$.

The fact that $\left(\pi_{i}\right)_{*} \circ \pi_{i}^{*}=[2]$ for $i=1,2$ already assures that $\operatorname{Jac}\left(F_{1}\right) \times \operatorname{Jac}\left(F_{2}\right)$ is isogenous to $\operatorname{Prym}(D / C)$. A quick way to see that they are actually isomorphic is by noting that $F_{1}$ and $F_{2}$ can be arbitrary hyperelliptic curves and that, by construction, the isogeny would have to depend functorially on $F_{1}$ and $F_{2}$. In general, $\operatorname{Jac}\left(F_{1}\right) \times \operatorname{Jac}\left(F_{2}\right)$ has no non-trivial polarization-preserving isogenies and hence there are no other candidates for $\operatorname{Prym}(D / C)$.

Remark 2.3. The result in Proposition 2.2 is easily extended to the situation where $f_{1}, f_{2}$ are quadratic conjugate over some quadratic extension $L$ over $K$. Then, over $L$ we still have $\operatorname{Jac}\left(F_{1}\right) \rightarrow$ $\operatorname{Prym}(D / C)$ and by taking Weil restrictions we see that $\Re_{L / K} \operatorname{Jac}\left(F_{1}\right)=\operatorname{Prym}(D / C)=\Re_{L / K}$ $\operatorname{Jac}\left(F_{2}\right)$.

Remark 2.4. In general, a hyperelliptic curve $C$ over $K$ is a double cover of a curve $L$ of genus 0 . We can express $K(L)$ as some quadratic extension of $K\left(\mathbb{P}^{1}\right)$. Relative Kummer theory allows us to describe $\operatorname{Prym}(D / C)$ in terms of Jacobians of subcovers of $D / L$ in exactly the same way as above.

\subsection{Linear subspaces on quadrics}

It is well known that on a non-singular quadric in $\mathbb{P}^{3}$, there are two rulings of lines with the property that a line from one ruling intersects a unique line from the opposite ruling and that two lines from the same ruling do not intersect. We need a simple lemma that classifies whether the two rulings are split or quadratic conjugates.

Lemma 2.5. Let $K$ be a field of characteristic different from two and let $M \in K^{4 \times 4}$ be a symmetric matrix describing a non-singular quadric $Q \subset \mathbb{P}^{3}$. The two rulings of lines on $Q$ are individually defined over $K$ exactly if $\operatorname{det}(M)$ is a square in $K$. Otherwise, they are quadratic conjugate.

Proof. First assume we have a point $\mathbf{x}_{0} \in Q(K)$. Let $V=\left\{\mathbf{x}: \mathbf{x}_{0}{ }^{t} M \mathbf{x}=0\right\}$ be the plane tangent to $Q$ at $\mathbf{x}$. The intersection $V \cap Q$ consists of exactly two lines through $\mathbf{x}_{0}$, one from each of the rulings. With a change of basis, we can assume that $\mathbf{x}_{0}=(1: 0: 0: 0)$ and that the points $\mathbf{x}_{1}=(0: 1: 0: 0)$ 
and $\mathbf{x}_{2}=(0: 0: 1: 0)$ lie on $V$ as well. It follows that

$$
M=\left(\begin{array}{cccc}
0 & 0 & 0 & d \\
0 & a & b & * \\
0 & b & c & * \\
d & * & * & *
\end{array}\right) ; \quad \operatorname{det}(M)=d^{2}\left(b^{2}-a c\right) .
$$

We see that $Q$ intersected with the line through $\mathbf{x}_{1}, \mathbf{x}_{2}$ is described by the equation $a x_{1}^{2}+2 b x_{1} x_{2}+$ $c x_{2}^{2}=0$, which is split exactly if $b^{2}-a c$ is a square. The lemma follows.

If $Q(K)$ is empty, then we base change to $K(Q)$, where we have the generic point $\left(x_{0}: x_{1}: x_{2}\right.$ : $\left.x_{3}\right) \in Q(K(Q))$. Since $K$ is algebraically closed in $K(Q)$, the pair of rulings (defined over $K$ ) is split over $K(Q)$ if and only if they are split over $K$. Furthermore, $\operatorname{det}(M)$ is a square in $K$ if and only if it is in $K(Q)$.

\section{Non-hyperelliptic curves of genus 5 with an unramified involution}

Let $K$ be a field of characteristic zero and let $D$ be a non-hyperelliptic curve of genus 5 with a fixed-point-free involution $\iota$ over $K$. Let $\pi: D \rightarrow C=D /\langle\iota\rangle$ be the quotient map by the action of $\iota$. The Riemann-Hurwitz formula yields that $C$ is of genus 3. Let $\kappa_{C}$ be a canonical divisor on $C$ and let $\langle u, v, w\rangle=\left|\kappa_{C}\right|$ be coordinates on the associated canonical model of $C$. Note that we do not insist that $C$ is non-hyperelliptic. By abuse of notation, we also write $u, v, w$ for the pull-backs $u \circ \pi, v \circ \pi, w \circ \pi$. We write $\kappa_{D}=\pi^{*}\left(\kappa_{C}\right)$, which is again canonical. There are functions $r, s$ on $D$ with $r+r \circ \iota=s+s \circ \iota=0$ such that $\langle u, v, w, r, s\rangle=\left|\kappa_{D}\right|$.

We identify $D$ with the canonical model associated to $\kappa_{D}$, being the image of $(u: v: w: r: s)$ : $D \rightarrow \mathbb{P}^{4}$. In this notation,

$$
\iota:(u: v: w: r: s) \mapsto(u: v: w:-r:-s) .
$$

We let $\Lambda$ be the linear system of quadrics containing $D \subset \mathbb{P}^{4}$. A simple comparison of the dimensions $l\left(\kappa_{D}\right)=5$ and $l\left(2 \kappa_{D}\right)=12$ yields that $\Lambda \simeq \mathbb{P}^{2}$. We let $\left(\lambda_{1}: \lambda_{2}: \lambda_{3}\right)$ be coordinates on $\Lambda$ such that $Q_{1}, Q_{2}, Q_{3} \in \Lambda(K)$ correspond to $(1: 0: 0)$, (0:1:0), (0:0:1), respectively.

Note that $\left|\kappa_{D}\right|$ has a decomposition into the +1 -eigenspace $\langle u, v, w\rangle$ and the -1-eigenspace $\langle r, s\rangle$ of $\iota$. The involution $\iota$ acts identically on the corresponding linear subspaces $\{r=s=0\},\{u=v=$ $w=0\} \subset \mathbb{P}^{4}$.

We recall that a curve is called trigonal if it admits a degree three map to $\mathbb{P}^{1}$.

Lemma 3.1. With the notation above, $D$ is not a trigonal curve.

Proof. Since a trigonal curve remains trigonal upon base extension, it suffices to prove the lemma for algebraically closed $K$. Suppose that $D$ were trigonal.

We argue following [ACGH85, p. 207]. The fibers of the degree three map $D \rightarrow \mathbb{P}^{1}$ form a basepoint-free linear system of degree three. Let $\mathfrak{D}$ be the divisor corresponding to one such fiber. Then $\mathfrak{D}$ is a $g_{3}^{1}$. Note that if $\mathfrak{D}$ were a $g_{3}^{2}$, then removing a point from $\mathfrak{D}$ would yield a $g_{2}^{1}$ and, hence, $D$ would be hyperelliptic, which we assumed not to be the case. Hence, $|\mathfrak{D}| \simeq \mathbb{P}^{1}$.

As is argued in [ACGH85, p. 207] (using the Brill-Noether residue theorem; see, for instance, [Ful69, ch. 8]), a curve of genus 5 can have at most one divisor class of type $g_{3}^{1}$. Therefore, $\iota_{*}$ : $\operatorname{Div}(D) \rightarrow \operatorname{Div}(D)$ induces an involution on $|\mathfrak{D}|$. Since an involution on $\mathbb{P}^{1}$ has two fixed points, there are two effective degree three divisors that are fixed under $\iota_{*}$. It follows that $\iota$ has a fixed point on $D$, which contradicts our assumption.

Lemma 3.2. With the notation above, $D=Q_{1} \cap Q_{2} \cap Q_{3}$. Furthermore, the $Q_{i}$ can be chosen to be non-singular and $D$ misses the singular locus of any quadric containing $D$. 


\section{N. BRUIN}

Proof. First note that if the statement of the lemma is false, then it is also false over the algebraic closure of $K$. Therefore, it suffices to prove the lemma for algebraically closed $K$. By Petri's theorem [ACGH85, p. 131], a canonical model of a non-hyperelliptic, non-trigonal curve of genus 5 is the intersection of quadrics.

Next we show that a quadric $Q \in \Lambda$ cannot be singular at $D$. Suppose that $P_{0} \in D$ is a singular point of some quadric $Q \in \Lambda$. Note that, if $\operatorname{rk} Q<3$, then there is a $\mathbb{P}^{3} \subset Q$. Since $D \subset Q$ too, we have that $D \cap \mathbb{P}^{3}$ is a curve. The space $\Lambda$ restricted to that $\mathbb{P}^{3}$ would be at most a pencil of quadrics and hence contain an intersection of two quadrics. This would imply that $D$ has a component of genus at most one, which contradicts that $D$ is a curve of genus 5 .

Hence, $Q$ is of rank three or four, which implies that $Q$ contains a $\mathbb{P}^{1}$ of planes through the singular point $P_{0} \in D$. On each such plane $V$, the restriction of $\Lambda$ is a pencil of conics and, hence, has a base locus of degree four. Since $P_{0} \in V \cap D$ is contained in that base locus, this realizes $D$ as a degree three cover of that $\mathbb{P}^{1}$. This contradicts that $D$ is non-trigonal.

By Bertini's theorem [Har77, Chapter III, Remark 10.9.2] it follows that a general member of $\Lambda$ is non-singular and hence we can choose the $Q_{i}$ to be non-singular.

Lemma 3.3. With the notation above, we have that $\iota$ acts trivially on $\Lambda$. Equivalently, for $Q \in \Lambda$, we can find quadratic forms $Q^{+} \in K[u, v, w]$ and $Q^{-} \in K[r, s]$ such that $Q$ is given by the equation

$$
Q^{+}(u, v, w)+Q^{-}(r, s)=0 .
$$

Proof. First note that $\iota$ preserves $D$ and, hence, preserves $\Lambda$. Suppose that there is $Q \in \Lambda(\bar{K})$ such that $Q \neq \iota(Q)$. Then $Q-\iota(Q) \in \Lambda(\bar{K})$ is not zero. On the other hand, since $\iota$ acts trivially on $\{r=s=0\}$, we have that $Q-\iota(Q)$ restricted to $\{r=s=0\}$ is zero. Hence, $\Lambda$ restricted to $\{r=s=0\}$ is a pencil of plane conics and has a base locus of degree four. This yields four points on $D$ that are fixed points of $\iota$, which contradicts the assumptions.

For an equation of any quadric in $\Lambda(\bar{K})$, this means that the monomials $u r, v r, \ldots, w s$ cannot occur.

\section{Special divisor classes of degree four}

As in the previous section, let $D$ be a non-hyperelliptic curve of genus 5 over a field $K$ of characteristic zero with an unramified involution $\iota: D \rightarrow D$. We also adopt the other notation from the previous section. We consider the scheme of special divisors

$$
W_{4}^{1}(D)=\left\{\mathcal{D} \in \operatorname{Pic}^{4}(D): l(\mathcal{D}) \geqslant 2\right\} .
$$

From the Riemann-Roch formula it follows that the residuation map $\mathcal{D} \mapsto\left[\kappa_{D}\right]-\mathcal{D}$ defines an involution on $W_{4}^{1}$.

We denote the locus of singular quadrics in $\Lambda$ by

$$
\Gamma=\{Q \in \Lambda: \operatorname{det}(Q)=0\} .
$$

By Lemma 3.3 we have a decomposition $\Gamma=\Gamma^{+} \cup \Gamma^{-}$, with equations

$$
\begin{aligned}
& \Gamma^{+}: \operatorname{det}\left(\lambda_{1} Q_{1}^{+}+\lambda_{2} Q_{2}^{+}+\lambda_{3} Q_{3}^{+}\right)=0, \\
& \Gamma^{-}: \operatorname{det}\left(\lambda_{1} Q_{1}^{-}+\lambda_{2} Q_{2}^{-}+\lambda_{3} Q_{3}^{-}\right)=0 .
\end{aligned}
$$

From the definition it is clear that $\Gamma$ is at least one-dimensional and, by Lemma 3.2, $\Gamma$ is at most one-dimensional too. In fact, it is straightforward to check that

$$
\Gamma^{\prime}=\{Q \in \Lambda: \operatorname{rk} Q=3\}
$$

is zero-dimensional and is the singular locus of $\Gamma$. 
Lemma 4.1. Let $\mathfrak{D}, \mathfrak{D}^{\prime} \in \operatorname{Div}(D)$ be effective divisors of degree four with $l(\mathfrak{D})=2$. Then the following hold.

(i) There is a unique 2-plane $V_{\mathfrak{D}}$ such that $\mathfrak{D}=D \cdot V_{\mathfrak{D}}$.

(ii) There is a unique quadric $Q_{\mathfrak{D}} \in \Lambda$ which vanishes on $V_{\mathfrak{D}}$. In fact, $Q_{\mathfrak{D}} \in \Gamma$.

(iii) If $V_{\mathfrak{D}}$ and $V_{\mathfrak{D}^{\prime}}$ meet in a line, then $\left[\mathfrak{D}+\mathfrak{D}^{\prime}\right]=\left[\kappa_{D}\right]$.

(iv) If $Q_{\mathfrak{D}}=Q_{\mathfrak{D}^{\prime}}$, then $\left[\mathfrak{D}^{\prime}+\mathfrak{D}\right]=\left[\kappa_{D}\right]$ or $\left[\mathfrak{D}-\mathfrak{D}^{\prime}\right]=0$.

(v) If $Q_{\mathfrak{D}} \neq Q_{\mathfrak{D}^{\prime}}$, then $\left[\mathfrak{D}^{\prime}+\mathfrak{D}\right] \neq\left[\kappa_{D}\right]$ and $\left[\mathfrak{D}-\mathfrak{D}^{\prime}\right] \neq 0$.

Proof. (i) The geometric formulation of the Riemann-Roch theorem [ACGH85, p. 12] states that, for a divisor $P_{1}+\cdots+P_{r}$ with $r \leqslant g$, we have

$$
l\left(P_{1}+\cdots+P_{r}\right)=r+1-\operatorname{rk}\left\langle P_{1}, \ldots, P_{r}\right\rangle,
$$

so one can take $V_{\mathfrak{D}}$ to be the plane spanned by the support of $\mathfrak{D}$ over $\bar{K}$.

(ii) Since the restriction of $\Lambda$ to $V_{\mathfrak{D}}$ has a base locus of degree four, it is a pencil of conics. Hence, there is a unique quadric $Q_{\mathfrak{D}} \in \Lambda$ that vanishes on $V_{\mathfrak{D}}$. Since a quadric in $\mathbb{P}^{4}$ containing a 2-plane is necessarily singular, it follows that $Q_{\mathfrak{D}} \in \Gamma$.

(iii) Two 2-planes meeting in a line lie in a 3-plane, say $W$. Since $\mathfrak{D}+\mathfrak{D}^{\prime}$ is effective of degree eight it equals the hyperplane section $D \cdot W$. Hyperplane sections of canonical models are canonical divisors. If $\mathfrak{D}$ and $\mathfrak{D}^{\prime}$ have intersecting support, then replace $\mathfrak{D}^{\prime}$ by a linearly equivalent effective divisor $\mathfrak{D}^{\prime \prime}$. The divisors $\mathfrak{D}^{\prime}$ and $\mathfrak{D}^{\prime \prime}$ certainly have disjoint support, since otherwise $\mathfrak{D}^{\prime}-\mathfrak{D}^{\prime \prime}$ would be the divisor of a function of degree at most three, contradicting Lemma 3.1.

(iv) First suppose that $Q_{\mathfrak{D}}$ is of rank four. Then $Q_{\mathfrak{D}}$ is a cone over a non-singular quadric in $\mathbb{P}^{3}$. The two line rulings on that quadric give rise to two plane 'rulings' on $Q_{\mathfrak{D}}$. Planes in opposite rulings meet in a line and planes in the same ruling meet only in the singular point of $Q_{\mathfrak{D}}$. Hence, if $V_{\mathfrak{D}}$ and $V_{\mathfrak{D}^{\prime}}$ belong to opposite rulings, then by part (iii) we have $\left[\mathfrak{D}+\mathfrak{D}^{\prime}\right]=\left[\kappa_{D}\right]$. If $V_{\mathfrak{D}}$ and $V_{\mathfrak{D}^{\prime}}$ belong to the same ruling, then both $\mathfrak{D}$ and $\mathfrak{D}^{\prime}$ are residual to an arbitrary divisor from the opposite ruling and, hence, linearly equivalent.

If $Q_{\mathfrak{D}}$ is of rank three, then $Q_{\mathfrak{D}}$ is a cone over a singular quadric in $\mathbb{P}^{3}$, so there is one ruling of planes on $Q_{\mathfrak{D}}$ and any two of these meet in a line. It follows by part (iii) that $\left[\mathfrak{D}+\mathfrak{D}^{\prime}\right]=\left[\kappa_{D}\right]$. Since this holds for any $\mathfrak{D}^{\prime}$ with $Q_{\mathfrak{D}^{\prime}}=Q_{\mathfrak{D}}$, it follows that $\left[\mathfrak{D}-\mathfrak{D}^{\prime}\right]=0$.

(v) Given $V_{\mathfrak{D}}$, there is a map

$$
\mathbb{P}^{1} \rightarrow\left\{\text { planes in } Q_{\mathfrak{D}}\right\}
$$

parametrizing the ruling on $Q_{\mathfrak{D}}$ containing $V_{\mathfrak{D}}$. Each of the planes $V$ in the image of this map cuts out an effective divisor $\mathfrak{D}^{\prime}$ equivalent to $\mathfrak{D}$. By construction, $Q_{\mathfrak{D}^{\prime}}=Q_{\mathfrak{D}}$. This gives an explicit realization of $\mathbb{P}^{1} \simeq|\mathfrak{D}|$, so this accounts for all divisors linearly equivalent to $\mathfrak{D}$.

Corollary 4.2. The map

$$
\begin{aligned}
\lambda: W_{4}^{1}(D) & \rightarrow \Gamma \\
{[\mathfrak{D}] } & \mapsto Q_{\mathfrak{D}}
\end{aligned}
$$

is well defined and realizes $W_{4}^{1}(D)$ as a double cover of $\Gamma$, ramified over $\Gamma^{\prime}$. The involution of $W_{4}^{1}(D)$ over $\Gamma$ corresponds to the residuation map $\mathcal{D} \mapsto\left[\kappa_{D}\right]-\mathcal{D}$.

\section{The Prym variety in genus 3}

We write $F$ for the union of components of $W_{4}^{1}(D)$ above $\Gamma^{-}$. If $\mathcal{D} \in F$, then $Q_{\mathcal{D}}$ has a singular point on $\{u=v=w=0\}$. If the plane $V_{\mathcal{D}}$ would not contain that singular point, then $Q_{\mathcal{D}}$ would 


\section{N. BRUIN}

contain a $\mathbb{P}^{3}$, but this contradicts that $\operatorname{rk}\left(Q_{\mathcal{D}}\right) \geqslant 3$ (by Lemma 3.2). Hence, $\pi\left(V_{\mathcal{D}}\right)$ is a line and $\pi_{*}(\mathcal{D})=\left[\kappa_{C}\right]$. It follows that the map

$$
\begin{array}{cccc}
j: & F \times F & \rightarrow & \operatorname{Pic}^{0}(D) \\
\mathcal{D}_{1}, \mathcal{D}_{2} & \mapsto & \mathcal{D}_{1}+\mathcal{D}_{2}-\left[\kappa_{D}\right]
\end{array}
$$

maps $F \times F$ into $\operatorname{ker}\left(\pi_{*}\right)$.

Depending on the type of $\Gamma^{-}$, this gives us different descriptions of $\operatorname{Prym}(D / C)$. The case numbering is in correspondence with Table 1.

Case 0: $\Gamma^{-}$is a doubled line. This case does not occur because assuming that it does, leads to a contradiction. By choosing coordinates appropriately, $\Gamma^{-}$is described by the equation $\lambda_{1}^{2}=0$. It follows that $Q_{1}^{-}+\lambda_{2} Q_{2}^{-}+\lambda_{3} Q_{3}^{-}$is non-singular for all $\lambda_{2}, \lambda_{3}$. It follows that $Q_{2}^{-}=Q_{3}^{-}=0$. Therefore, $D$ has an intersection with $\{u=v=w=0\}$ and thus $\iota$ would be ramified.

Case 2: $\Gamma^{-}$is a split singular conic, i.e. $L_{1} \cup L_{2}$, for lines $L_{1}, L_{2}$ over $K$. In that situation, $F$ consists of two components $E_{1}$ and $E_{2}$, covering $L_{1}$ and $L_{2}$, respectively. Each of these covers is ramified at a degree four locus: the intersection of $L_{i}$ with the other components of $\Gamma$. Hence, $E_{1}$ and $E_{2}$ are curves of genus 1 . In fact, each has a rational point above $L_{1} \cap L_{2}$, so we can identify them with their Jacobians. We have the map

$$
\begin{aligned}
E_{1} \times E_{2} & \rightarrow \operatorname{Jac}(D) \\
\left(\mathcal{D}_{1}, \mathcal{D}_{2}\right) & \mapsto \mathcal{D}_{1}+\mathcal{D}_{2}-\left[\kappa_{D}\right] .
\end{aligned}
$$

Note that $\left(\mathcal{D}_{1}, \mathcal{D}_{2}\right)$ only maps to $O$ if $Q_{\mathcal{D}_{1}}=Q_{\mathcal{D}_{2}}$. This can only happen above the (ramified) point $L_{1} \cap L_{2}$, so the map is an injection. Since $E_{1} \times E_{2}$ is connected and contains the origin, the image is contained in $\operatorname{Prym}(D / C)$ and because $E_{1} \times E_{2}$ is an Abelian surface itself, we have the equality

$$
\operatorname{Prym}(D / C) \cong E_{1} \times E_{2} .
$$

Case 3: $\Gamma^{-}$is a non-split singular conic. Then, over some quadratic extension $K(\sqrt{d})$ of $K$, the conic $\Gamma^{-}$splits and the analysis above applies. It follows that in that situation $E_{1}$ and $E_{2}$ are elliptic curves that are conjugate with respect to $K(\sqrt{d}) / K$. By Weil restriction, it follows that

$$
\operatorname{Prym}(D / C) \cong \Re_{K(\sqrt{d}) / K}\left(E_{1}\right) .
$$

Case 4: $\Gamma^{-}$is a non-singular conic. In that case, $Q_{1}^{-}, Q_{2}^{-}, Q_{3}^{-}$are $K$-linearly independent and therefore span the space of quadratic forms in $r, s$. Without loss of generality, we can assume

$$
\begin{aligned}
& Q_{1}: Q_{1}^{+}(u, v, w)=r^{2}, \\
& Q_{2}: Q_{2}^{+}(u, v, w)=r s, \\
& Q_{3}: Q_{3}^{+}(u, v, w)=s^{2} .
\end{aligned}
$$

It follows that $\Gamma^{-}$is given by the equation $4 \lambda_{1} \lambda_{3}=\lambda_{2}^{2}$ and we have a parametrization

$$
\begin{array}{ccc}
\mathbb{P}^{1} & \rightarrow & \Gamma^{-} \\
(x: 1) & \mapsto & \left(1: 2 x: x^{2}\right) .
\end{array}
$$

The curve $F$ is a double cover of $\Gamma^{-}$, ramified above $\Gamma^{+} \cap \Gamma^{-}$. Using the parametrization above we obtain an equation of the form

$$
\operatorname{det}\left(Q_{1}^{+}+2 x Q_{2}^{+}+x^{2} Q_{3}^{+}\right)=\delta y^{2}
$$

for some $\delta \in K^{*}$. Note that $\Gamma^{+} \cap \Gamma^{-}$has no multiple points, since those would correspond to conics in $\Lambda$ of rank smaller than three, which contradicts Lemma 3.2. It follows that $F$ is branched over six points and, hence, is of genus 2 . 


\section{The ARIThmetic of Prym VARIETIES IN GENUS 3}

TABLE 1. Structure of the Prym variety.

\begin{tabular}{ccccc}
\hline Case & $C$ & $D$ & $\Gamma^{-}$ & $\operatorname{Prym}(D / C)$ \\
\hline 1 & Hyperelliptic & Hyperelliptic & - & $\operatorname{Jac}(F)$ \\
2 & Hyperelliptic & Non-hyperelliptic & Split singular & $E_{1} \times E_{2}$ \\
3 & Hyperelliptic & Non-hyperelliptic & Non-split singular & $\Re_{K(\sqrt{d}) / K}(E)$ \\
4 & Non-hyperelliptic & Non-hyperelliptic & Non-singular & $\operatorname{Jac}(F)$ \\
\hline
\end{tabular}

In order to determine the correct value of $\delta$, suppose that we have $\mathcal{D} \in F$ such that $2 \mathcal{D} \neq\left[\kappa_{D}\right]$. Then $\mathcal{D}$ is cut out by a system of planes on $Q=\lambda(\mathcal{D})$, as defined in Corollary 4.2. If $\mathcal{D}$ is rational over $K$, then $Q$ and the system of planes must be as well (but note that $\mathcal{D}$ itself does not need to contain rational divisors, nor does the system need to contain any planes rational over $K$ ).

If no rational $\mathcal{D}$ is available, then we base extend to $K(F)$ to ensure there is. We only want to determine the value of $\delta$ modulo squares and since $K$ is algebraically closed in $K(F)$, our results will be valid as long as we find $\delta \in K$.

Using the parametrization of $\Gamma^{-}$, there is an $(x: 1) \in \mathbb{P}^{1}(K)$ such that

$$
Q^{-}=-\left(r^{2}+2 x r s+x^{2} s^{2}\right) \text {. }
$$

It follows that $(0: 0: 0: x:-1)$ is the singular point of $Q$ and that $Q$ is a cone over the quadric in $\mathbb{P}^{3}=\{s=0\} \subset \mathbb{P}^{4}$ with coordinates $(u: v: w: r)$ given by

$$
Q^{+}(u, v, w)-r^{2}=0 .
$$

According to Lemma 2.5, this quadric has two rational systems of lines (and, therefore, a cone over it has two rational systems of planes) if

$$
-\operatorname{det} Q^{+}=-\operatorname{det}\left(Q_{1}^{+}+2 x Q_{2}^{+}+x^{2} Q_{3}^{+}\right)
$$

is a square in $K$. It follows that $F$ is isomorphic to

$$
F: y^{2}=-\operatorname{det}\left(Q_{1}^{+}+2 x Q_{2}^{+}+x^{2} Q_{3}^{+}\right) .
$$

The map $j: F \times F \rightarrow \operatorname{Pic}^{0}(D)$ defined in (2) gives rise to an isomorphism

$$
\operatorname{Prym}(D / C) \simeq \operatorname{Jac}(F) \text {. }
$$

In Cases 2 and 3 above, the Jacobian of $D$ contains elliptic curves $E_{1}, E_{2}$. In these cases, $D$ is in fact a double cover of genus 1 curves $C_{1}$ and $C_{2}$ with $\operatorname{Jac}\left(C_{i}\right) \cong E_{i}$.

The cover can be constructed explicitly in the following way. A linear system of singular quadrics has a fixed singularity. Therefore, the $Q \in L_{i} \subset \Gamma^{-}$have a fixed singularity $P_{i}$ on the line $\{u=v=$ $w=0\}$. When we project $D$ from $P_{i}$, we obtain an intersection of two quadrics in $\mathbb{P}^{3}$. Thus, this presents $D$ as a double cover of a genus 1 curve $C_{i}$.

Let $\sigma_{i} \in \operatorname{Aut}_{\bar{K}}(D)$ denote the involution of $D$ over $C_{i}$. We have $\iota=\sigma_{1} \sigma_{2}$. In fact, the projection $(u: v: w: r: s) \rightarrow(u: v: w)$ corresponds to $D \rightarrow D /\left\langle\sigma_{1}, \sigma_{2}\right\rangle$. This shows that the canonical model of $C$ is, in fact, of genus 0 and, hence, that $C$ is hyperelliptic. This places us in the situation of $\S 2.2$.

Theorem 5.1. Let $K$ be a field of characteristic zero and let $C$ be a curve of genus 3 over $K$ with an unramified double cover $D / C$. Then $\operatorname{Prym}(D / C)$ can be described as given in Table 1 depending on the nature of $C$ and $D$. Models of the curves involved can be described as in Table 2. For hyperelliptic curves, it is assumed they are hyperelliptic over a $\mathbb{P}^{1}$ over $K$. 


\section{N. BRUIN}

TABLE 2. Models of involved curves.

\begin{tabular}{|c|c|}
\hline Case & Models \\
\hline 1 & $\begin{array}{ll}C: & y^{2}=Q(x) R(x) \text { where } \operatorname{deg}(Q)=2, \operatorname{deg}(F)=6 \\
D: & y_{1}^{2}=Q(x) \text { and } y_{2}^{2}=R(x) \\
F: & y_{2}^{2}=R(x)\end{array}$ \\
\hline 2 & $\begin{array}{ll}C: & y^{2}=R_{1}(x) R_{2}(x) \text { where } \operatorname{deg}\left(R_{1}\right)=\operatorname{deg}\left(R_{2}\right)=4 \\
D: & y_{1}^{2}=R_{1}(x) \text { and } y_{2}^{2}=R_{2}(x) \\
E_{1}: & \operatorname{Jac}\left(y_{1}^{2}=R_{1}(x)\right) \\
E_{2}: & \operatorname{Jac}\left(y_{2}^{2}=R_{2}(x)\right)\end{array}$ \\
\hline 3 & $\begin{array}{ll}C: & y^{2}=N_{K(\sqrt{d})[x] / K[x]} R(x) \text { where } \operatorname{deg}(R)=4 \\
D: & \left(x, y_{0}, y_{1}\right) \text { satisfying }\left(y_{0}+y_{1} \sqrt{d}\right)^{2}=R(x) \\
E: & \operatorname{Jac}\left(y^{2}=R(x)\right)\end{array}$ \\
\hline 4 & $\begin{array}{ll}C: & Q_{1}^{+}(u, v, w) Q_{3}^{+}(u, v, w)=Q_{2}^{+}(u, v, w)^{2} \text { with } Q_{i}^{+} \text {quadratic forms } \\
D: & \left\{\begin{array}{l}Q_{1}^{+}(u, v, w)=r^{2} \\
Q_{2}^{+}(u, v, w)=r s \\
Q_{3}^{+}(u, v, w)=s^{2}\end{array}\right. \\
F: & y^{2}=-\operatorname{det}\left(Q_{1}^{+}+2 x Q_{2}^{+}+x^{2} Q_{3}^{+}\right)\end{array}$ \\
\hline
\end{tabular}

\section{Mapping $D$ into $\operatorname{Prym}(D / C)$}

As was shown in $\S 5$, if $C$ is hyperelliptic, then $D$ is a cover of the curves that $\operatorname{span} \operatorname{Prym}(D / C)$. Hence, it is obvious how to map $D$ into $\operatorname{Prym}(D / C)$. In this section we show how $D$ can be mapped into $\operatorname{Prym}(D / C)$ if $C$ is non-hyperelliptic.

First, if we have a rational point $P_{0} \in D(K)$, we can embed $D$ in $\operatorname{Jac}(D)$ via the Abel-Jacobi map

$$
\begin{aligned}
& D \rightarrow \operatorname{Jac}(D) \\
& P \mapsto\left[P-P_{0}\right] .
\end{aligned}
$$

When we combine this map with the projection map $\left(\mathrm{id}_{*}-\iota_{*}\right): \operatorname{Jac}(D) \rightarrow \operatorname{Prym}(D / C)$, we obtain the Abel-Prym map

$$
\begin{aligned}
& D \rightarrow \operatorname{Prym}(D / C) \\
& P \mapsto[P-\iota(P)]-\left[P_{0}-\iota\left(P_{0}\right)\right] .
\end{aligned}
$$

In general, we do not have a rational base point $P_{0}$ at our disposal. We give an alternative map, based on the description of $\operatorname{Prym}(D / C)$ as $\operatorname{Jac}(F)$ for some component $F$ of $W_{4}^{1}(D)$. This map turns out to correspond to $2\left(\iota_{*}-\mathrm{id}_{*}\right): \operatorname{Pic}^{1}(D) \rightarrow \operatorname{Prym}(D / C)$. Thus, the image of $D$ is a translation of twice an Abel-Prym embedding of $D$.

Let $P_{0}$ be a point on $D$ and let $L$ be the tangent line of $C$ at $\pi(P)$. Then $L \cdot C$, being a linear section of a canonical model, determines an effective canonical divisor on $C$. Consequently, for some $P_{1}, P_{2} \in D$ we have that $\pi^{*}(L \cdot C)=2 P_{0}+2 \iota\left(P_{0}\right)+P_{1}+\iota\left(P_{1}\right)+P_{2}+\iota\left(P_{2}\right)$ is an effective canonical divisor on $D$.

We use the notation from $\S 3$. Furthermore, we write $\operatorname{Tang}_{D}(P)$ for the tangent line of $D$ at $P$ and for a quadric $Q \subset \mathbb{P}^{n}$ and $T_{1}, T_{2} \in \mathbb{P}^{n}$ we write $T_{1}^{t} Q T_{2}$ for the matrix product, where $Q$ is identified with its representing $(n+1) \times(n+1)$ symmetric matrix and $T_{1}, T_{2}$ are interpreted as $(n+1)$-dimensional column vectors of projective coordinates. 
Lemma 6.1. With the notation as above, let $P$ be a point in $D(\bar{K})$.

(i) There are two divisors $\mathfrak{D}_{1}, \mathfrak{D}_{2}$ on $D$ (with $\mathfrak{D}_{1}=\mathfrak{D}_{2}$ in degenerate cases) such that $\mathfrak{D}_{i} \geqslant 2 P$ and $\left[\mathfrak{D}_{i}\right] \in F(\bar{K}) \subset W_{4}^{1}(\bar{K})$.

(ii) Let $P_{1}, P_{2}$ be the points on $D$ such that $\mathfrak{D}_{1}=2 P+P_{1}+P_{2}$. Then $\mathfrak{D}_{2}=2 P+\iota\left(P_{1}\right)+\iota\left(P_{2}\right)$.

(iii) Let $T \in \operatorname{Tang}_{D}(P)$ with $T \neq P$. If $\mathfrak{D} \in F$ with $D=2 P+P_{1}+P_{2}$, then $x=x_{*}(\mathfrak{D})$ satisfies

$$
\left(T^{t} \cdot Q_{1} \cdot T\right)+2 x\left(T^{t} \cdot Q_{2} \cdot T\right)+x^{2}\left(T^{t} \cdot Q_{3} \cdot T\right)=0 .
$$

(iv) The map

$$
\begin{aligned}
\varphi: D(\bar{K}) & \rightarrow \operatorname{Div}^{2}(F / \bar{K}) \\
P & \mapsto\left[\mathfrak{D}_{1}\right]+\left[\mathfrak{D}_{2}\right]
\end{aligned}
$$

is defined over $K$.

Proof. (iii) Note that $\mathfrak{D} \in F$ implies $l(\mathfrak{D})=2$. By Lemma 4.1 we have $\operatorname{Tang}_{D}(P) \subset V_{\mathfrak{D}} \subset Q_{\mathfrak{D}}$. This implies that $T \in Q_{\mathfrak{D}}$ for some (and, hence, for all) $T \in \operatorname{Tang}_{D}(P)$ with $T \neq P$. Using that $\Gamma^{-} \simeq \mathbb{P}^{1}$ via $\left(1: 2 x: x^{2}\right) \mapsto(x: 1)$, we get the desired equation.

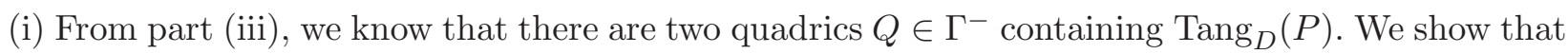
such a quadric is $Q_{\mathfrak{D}}$ for some $\mathfrak{D} \in F$ such that $\mathfrak{D} \geqslant 2 P$. Consider the plane $V$ spanned by $\operatorname{Tang}_{D}(P)$ and a singular point $(0: 0: 0: x:-1)$ of $Q$. Then $V \subset Q$. The proof of Lemma 4.1(v) points out that then $\mathfrak{D}=V \cdot D$ represents a divisor class in $W_{4}^{1}(D)$ and that $Q=Q_{\mathfrak{D}}$. By construction, we have $\mathfrak{D} \geqslant 2 P$.

If there is another divisor $\mathfrak{D}^{\prime} \geqslant 2 P$ with $Q_{\mathfrak{D}^{\prime}}=Q$, then $\mathfrak{D}-\mathfrak{D}^{\prime}$ or $\mathfrak{D}-\iota_{*}\left(\mathfrak{D}^{\prime}\right)$ is principal. Taking the image under $\pi_{*}$ would give a divisor of a degree two function on $C$, which contradicts that $C$ is not hyperelliptic.

(ii) Note that $\pi_{*}\left(\mathfrak{D}_{2}\right)=\pi_{*}\left(\mathfrak{D}_{1}\right)=C \cdot \operatorname{Tang}_{C}(\pi(P))$. If $\mathfrak{D}_{2}=2 P+P_{1}+\iota\left(P_{2}\right)$, then $2 P+P_{1}$ must lie on the line $V_{\mathfrak{D}_{1}} \cap V_{\mathfrak{D}_{2}}$. Since $D$ is canonical, it follows that $l\left(2 P+P_{1}\right)=2$. This contradicts Lemma 3.1. It follows that $\mathfrak{D}_{2}$ must be as stated.

(iv) Verify that ${ }^{\sigma}(\varphi(P))=\varphi\left({ }^{\sigma} P\right)$ for $\sigma \in \operatorname{Gal}(\bar{K} / K)$ via direct computation.

Proposition 6.2. Let $C$ be a non-hyperelliptic genus 3 curve over a field $K$ of characteristic zero and let $D / C$ be an unramified double cover with $\iota: D \rightarrow D$ the associated involution. Let $F$ be the genus 2 curve given by Theorem 5.1 such that $\operatorname{Jac}(F)=\operatorname{Prym}(D / C)$ and let $\varphi: D \rightarrow \operatorname{Div}^{2}(F)$ be the map defined in Lemma 6.1. Then we have

$$
\begin{aligned}
2\left(\iota_{*}-\mathrm{id}_{*}\right): D(\bar{K}) & \rightarrow & \operatorname{Prym}(D / C)(\bar{K}) \\
P & \mapsto & {\left[\varphi(P)-\kappa_{F}\right] }
\end{aligned}
$$

Proof. Using Lemma 6.1(ii), we have $\varphi(P)=4 P+P_{1}+P_{2}+\iota\left(P_{1}\right)+\iota\left(P_{2}\right)$. Using that $\left[\kappa_{D}\right]=$ $\left[2 P+2 \iota(P)+P_{1}+P_{2}+\iota\left(P_{1}\right)+\iota\left(P_{2}\right)\right]$, we have $\left[\varphi(P)-\kappa_{D}\right]=2 P-2 \iota(P)$ as an element of Pic $(D)$. Note that $\left[\kappa_{D}\right]=[\mathfrak{D}+\iota(\mathfrak{D})]$ for any $[\mathfrak{D}] \in F \subset W_{4}^{1}(D)$, so identifying $\operatorname{Pic}(F) \subset \operatorname{Pic}(D)$, we get $\left[\kappa_{F}\right]=\left[\kappa_{D}\right]$. This proves the proposition.

Remark 6.3. It is worth noting that the map $\left(\iota_{*}-\mathrm{id}_{*}\right): D \rightarrow \operatorname{ker}\left(\pi_{*}\right)$ does not map $D$ to $\operatorname{Prym}(D / C)$. To see this, note that $\operatorname{Prym}(D / C)=\operatorname{Jac}(F)$ for some genus 2 curve $F$. Any degree zero divisor class on $F$ can be represented as the difference of two points on $F$, so we have that $\operatorname{Prym}(D / C)=F-F$. If $P \in D(\bar{K})$ has $[\iota(P)-P] \in \operatorname{Prym}(D / C)$, then we can find $\left[\mathfrak{D}_{1}\right],\left[\mathfrak{D}_{2}\right] \in F(\bar{K})$ such that $[\iota(P)-P]=$ $\left[\mathfrak{D}_{1}-\mathfrak{D}_{2}\right]$. Since $F \subset W_{4}^{1}(D)$, we can choose effective representatives $\mathfrak{D}_{1}, \mathfrak{D}_{2}$ with a given point in the support. Hence, we can assume that $\mathfrak{D}_{1}=\iota(P)+P_{2}+P_{3}+P_{4}$ and $\mathfrak{D}_{2}=P+P_{5}+P_{6}+P_{7}$. Note that $P_{2}, P_{3}, P_{4}$ are not collinear, because if they were, then the geometric formulation of the 


\section{N. BRUIN}

Riemann-Roch theorem [ACGH85, p. 12] would imply that $l\left(P_{2}+P_{3}+P_{4}\right)=2$, so $D$ would be trigonal or hyperelliptic.

Therefore, $P_{2}, P_{3}, P_{4}$ already determine the plane $V_{\mathfrak{D}_{1}}$. If $P_{5}+P_{6}+P_{7}=P_{2}+P_{3}+P_{4}$, then we would have that both $P$ and $\iota P$ lie in the same plane $V_{\mathfrak{D}_{1}}$, which would yield a $g_{5}^{2}$ on $D$. However, then the Riemann-Roch theorem tells us that $\kappa_{D}-P-\iota(P)-P_{2}-P_{3}-P_{4}$ would be a $g_{3}^{1}$ and therefore $D$ would be trigonal.

It follows that the non-zero divisor $P_{2}+P_{3}+P_{4}-P_{5}-P_{6}-P_{7}$ is linearly equivalent to zero, so $D$ is hyperelliptic or trigonal, contradicting Lemma 3.1 .

Lemma 6.1 together with Proposition 6.2 provide an explicit way of mapping $D$ into an Abelian surface $\operatorname{Prym}(D / C) \simeq \operatorname{Jac}(F)$. By slight abuse of notation, we write $\varphi: D \rightarrow \operatorname{Jac}(F)$.

For explicit computations, Abelian surfaces have proven to be rather unwieldy. In many cases, enough of the group variety structure remains in the associated Kummer surface $\mathcal{K}=\operatorname{Jac}(F) /\langle-1\rangle$. The surface $\mathcal{K}$ is naturally expressed as a quartic surface in $\mathbb{P}^{3}$. We use the map ${ }^{2}$ from $[\mathrm{CF} 96,3.1]$

$$
\begin{aligned}
& k: \quad \operatorname{Jac}(F) \quad \rightarrow \quad \mathcal{K} \\
& {\left[\left(x_{1}, y_{1}\right)+\left(x_{2}, y_{2}\right)-\kappa_{F}\right] \mapsto\left(1: x_{1}+x_{2}: x_{1} x_{2}: \ldots\right)=\left(k_{1}: k_{2}: k_{3}: k_{4}\right)}
\end{aligned}
$$

which expresses $\operatorname{Jac}(F)$ as a double cover of $\mathcal{K}$, ramified at $\operatorname{Jac}(F)[2]$, which maps to the singular locus of $\mathcal{K}$. The equation of $\mathcal{K}$ is of the form

$$
\mathcal{K}:\left(k_{2}^{2}-4 k_{1} k_{3}\right) k_{4}^{2}+K_{3}\left(k_{1}, k_{2}, k_{3}\right) k_{4}+K_{4}\left(k_{1}, k_{2}, k_{3}\right)=0,
$$

where $K_{3}$ and $K_{4}$ are homogeneous forms of degrees three and four, respectively (see [CF96] for explicit formulae). Hence, $\mathcal{K}$ is itself a double cover of the projective plane with coordinates $\left(k_{1}\right.$ : $\left.k_{2}: k_{3}\right)$ outside the point $(0: 0: 0: 1)$.

Since $\iota_{*} \circ 2\left(\iota_{*}-\mathrm{id}_{*}\right)=-1 \circ 2\left(\iota_{*}-\mathrm{id}_{*}\right)$, we see that $D \rightarrow k \varphi(D)$ factors through $D /\langle\iota\rangle=C$. Furthermore, if $\mathfrak{D} \in \operatorname{Div}^{2}(F)$ is effective and $\left(k_{1}: k_{2}: k_{3}: k_{4}\right)=k\left(\left[\mathfrak{D}-\kappa_{F}\right]\right)$, then $x_{*}(\mathfrak{D})$ satisfies

$$
k_{3}-k_{2} x+k_{1} x^{2}=0 .
$$

This gives us a procedure to compute many pointwise images for $k \varphi$.

(1) Choose an extension $L$ and a point $P \in D(L)$ (since $D$ is given as a degree eight curve, there is an abundance of suitable degree eight extensions).

(2) Following Lemma 6.1, choose $T \in \operatorname{Tang}_{D}(P)$ and set

$$
\left(k_{1}, k_{2}, k_{3}\right)=\left(T^{t} \cdot Q_{1} \cdot T,-2 T^{t} \cdot Q_{2} \cdot T, T^{t} \cdot Q_{3} \cdot T\right) .
$$

(3) If $k_{3}-k_{2} x+k_{1} x^{2}$ is irreducible of degree two over $L$, then there is a unique point $\left(k_{1}: k_{2}:\right.$ $\left.k_{3}: k_{4}\right) \in \mathcal{K}(L)$ that has an $L$-rational preimage in $\operatorname{Jac}(F)$. This is the desired image.

The irreducibility in the last step corresponds to $P_{1}, P_{2}$ from Lemma 6.1(ii) being quadratic conjugate over $L$. In that case, the divisor $P_{1}+\iota\left(P_{2}\right)$ is not $L$-rational and, hence, rationality tells which divisor to pick. If $P_{1}, P_{2}$ are themselves $L$-rational, then the above procedure does not compute sufficient information to distinguish between $2 P+P_{1}+P_{2}$ and $P+\iota(P)+P_{1}+\iota\left(P_{2}\right)$.

The procedure above yields a way to compute the equations of $k \varphi(D)$. First, one gathers many pointwise images for points over extensions $L$ and then one interpolates for low degree rational forms vanishing on those points. As we will see, $k \varphi(D)$ is the intersection of $\mathcal{K}$ with another degree four surface.

${ }^{2}$ If $F$ is given by the equation $y^{2}=f_{0}+f_{1} x+f_{2} x^{2}+\cdots+f_{6} x^{6}$, then

$$
k_{4}=\frac{2 f_{0}+f_{1} k_{2}+2 f_{2} k_{3}+f_{3} k_{2} k_{3}+2 f_{4} k_{3}^{2}+f_{5} k_{2} k_{3}^{2}+2 f_{6} k_{3}^{3}-2 y_{1} y_{2}}{\left(x_{1}-x_{2}\right)^{2}} .
$$




\section{The ARITHMetic OF Prym VARIETIEs IN GenUs 3}

Lemma 6.4. With the notation above, the image of $D$ under $k \circ \varphi$ is of degree at most 16 .

Proof. We compute the degree of the image by computing the degree of the intersection with $k_{1}=0$. By change of basis we can assume that $Q_{3}$ is of rank four. According to Lemma 6.1(iii), a point $P \in D$ has $k_{1}=0$ if $Q_{3}$ contains the plane $V_{\mathfrak{D}}$ for some $\mathfrak{D} \geqslant 2 P$. The two plane rulings on $Q_{3}$ give rise to two degree four covers $D \rightarrow \mathbb{P}^{1}$, where the fibres are the divisors cut out by the $V_{\mathfrak{D}}$ in the ruling. From Riemann-Hurwitz it follows that for each ruling there are 16 ramified fibres, i.e. $\mathfrak{D}$ of the form $2 P+P_{1}+P_{2}$. Hence, we see that there are 32 points on $D$ (counted with appropriate multiplicity) that land on $k_{1}=0$. Note that the image of $D$ under $k \varphi$ factors through $D /\langle\iota\rangle$, so the degree of $k \varphi(D)$ is at most 16 .

The procedure above has been implemented as a routine for the computer algebra system MAGMA [BCP97]. See [Bru04].

\section{The fibre of the Prym map in genus 3}

Given a genus 2 curve $F$, we have an Abelian variety $\operatorname{Jac}(F)$ and a quartic surface $\operatorname{Jac}(F) /\langle-1\rangle=$ $\mathcal{K} \subset \mathbb{P}^{3}$, with a singular locus consisting of the image of $\operatorname{Jac}(F)[2]$. There is an obvious way of realizing $\operatorname{Jac}(F)$ as a Prym variety of a non-hyperelliptic curve of genus 3. Pick a plane $V \subset \mathbb{P}^{3}$ such that $C:=V \cap \mathcal{K}$ is a non-singular quartic curve. It follows that $C$ stays away from the singular points of $\mathcal{K}$ and thus does not meet the ramification locus of $k: \operatorname{Jac}(F) \rightarrow \mathcal{K}$. Therefore, $D=k^{-1}(C)$ is an unramified cover of $C$. Either $D$ is connected and, hence, of genus 5 or $D$ is the disjoint union of two copies of $C$. Note, however, that $C$ is of genus 3 and, hence, has to be special to fit in an Abelian surface.

In fact, as Verra [Ver87] proves, over an algebraically closed field, essentially any occurrence of $\operatorname{Jac}(F)$ as $\operatorname{Prym}(D / C)$ occurs for $C$ isomorphic to a linear section of $\mathcal{K}$. The addition of $\operatorname{Jac}(F)[2]$ induces automorphisms of $\mathcal{K}$ which are induced by linear transformations of $\mathbb{P}^{3}$. He shows that the fibre of the Prym map $(D / C) \mapsto \operatorname{Prym}(D / C)$ over $\operatorname{Jac}(F)$ is a blow-up of $\widehat{\mathbb{P}}^{3} / \operatorname{Jac}(F)[2]$, where $\widehat{\mathbb{P}}^{3}$ is the space of plane sections of $\mathcal{K}$.

Verra also proves that genus 5 curves $D \subset \operatorname{Jac}(F)$ of the form above are, up to translation by a 2-torsion point, Abel-Prym embeddings. We give a short description of a procedure to recover

$$
C: Q_{1}^{+}(u, v, w) Q_{3}^{+}(u, v, w)=Q_{2}^{+}(u, v, w)^{2}
$$

from a plane section of the $\operatorname{Kummer}$ surface $\mathcal{K}=\operatorname{Jac}(F) /\langle-1\rangle$ for a curve $F$ of genus 2 .

First we review some of the basic geometry of Jacobians of curves of genus 2. Let $F$ be a curve of genus 2. In [CF96], Cassels and Flynn define a projective model of $\operatorname{Jac}(F)$ in $\mathbb{P}^{15}$ with coordinates $\left(z_{0}: \cdots: z_{15}\right)$ with (among others) the following properties.

- There is a symmetric theta divisor $\Theta$ on $\operatorname{Jac}(F)$ such that

$$
\left\langle k_{1}, \ldots, k_{4}\right\rangle=|2 \Theta|
$$

and

$$
\left\langle z_{0}, \ldots, z_{15}\right\rangle=|4 \Theta|
$$

with

$$
\left(k_{1}: k_{2}: k_{3}: k_{4}\right)=\left(z_{14}: z_{13}: z_{12}: z_{5}\right) .
$$

- The coordinates $\left(k_{1}: k_{2}: k_{3}: k_{4}\right)$ provide a model of the Kummer surface $\mathcal{K}=\operatorname{Jac}(F) /\langle-1\rangle$.

- With respect to the action of $[-1] \in \operatorname{Aut}(\operatorname{Jac}(F))$ on $|4 \Theta|$, we have that

$$
\left\langle z_{0}, z_{3}, z_{4}, z_{5}, z_{10}, \ldots, z_{15}\right\rangle=\left\langle k_{1}^{2}, k_{1} k_{2}, \ldots, k_{4}^{2}\right\rangle
$$




\section{N. BRUIN}

is the +1 -eigenspace and

$$
\left\langle g_{0}, \ldots, g_{5}\right\rangle:=\left\langle z_{1}, z_{2}, z_{6}, z_{7}, z_{8}, z_{9}\right\rangle
$$

is the -1-eigenspace.

Let $V=\left\{k_{4}=v_{1} k_{1}+v_{2} k_{2}+v_{3} k_{3}\right\} \subset \mathbb{P}^{3}$ be a plane such that $C=\mathcal{K} \cap V$ is a non-singular plane section and let $(u: v: w)=\left(k_{1}: k_{2}: k_{3}\right)$ be coordinates on $V$ (since $(0: 0: 0: 1)$ is a singular point of $\mathcal{K}$, assuming this suggested form of $V$ is not a restriction). On $V$, we have that $k_{4}=k_{4}(u, v, w)$ is a linear form in $u, v, w$. The curve $C$ is a non-singular degree four plane curve. It follows that $C$ is of genus 3 and that $(u: v: w)$ gives a canonical model of $C$, i.e. that $\left\langle\left. u\right|_{C},\left.v\right|_{C},\left.w\right|_{C}\right\rangle=\left|\kappa_{C}\right|$ for some canonical divisor $\kappa_{C}$ of $C$. Let $D$ be the pull-back of $C$ along $\operatorname{Jac}(F) \rightarrow \mathcal{K}$ and let $\kappa_{D}$ be the pull-back of $\kappa_{C}$. It is a straightforward computation to check that the restriction of $\left\langle z_{0}, \ldots, z_{15}\right\rangle$ to $D$ gives a linear system contained in $\left|\kappa_{D}\right|$ and that generically it gives the complete linear system.

Using the quadratic relations between the $z_{i}$ (see [CF96], [Fly96] and [Fly90] for the explicit formulae), we can express any $g_{i} g_{j}$ as a degree four form in $k_{1}, \ldots, k_{4}$. Hence, we obtain that

$$
\left(a_{0} g_{0}+\cdots+a_{5} g_{5}\right)^{2}=G\left(a_{0}, \ldots, a_{5} ; k_{1}, \ldots, k_{4}\right),
$$

where $G$ is homogeneous of degrees two and four in the $a_{i}$ and the $k_{j}$, respectively.

Insisting that

$$
G\left(a_{0}, \ldots, a_{5} ; u, v, w, k_{4}(u, v, w)\right)=u^{2} Q(u, v, w)
$$

for some quadratic form $Q$ gives nine quadratic equations in $a_{0}, \ldots, a_{5}$. However, a solution to these equations corresponds exactly to a form $Q(u, v, w)$ on $C$ that becomes a square when pulled back to $D$. We know that this happens for exactly two forms $Q_{1}^{+}(u, v, w)=r^{2}$ and $Q_{3}^{+}(u, v, w)=s^{2}$, so these equations determine a degree two locus in $\left(a_{0}: \cdots: a_{5}\right)$.

Solving these equations allows us to determine $Q_{1}^{+}(u, v, w)$ and $Q_{3}^{+}(u, v, w)$ up to a scalar. The quadratic form $Q_{2}^{+}(u, v, w)$ is then easily determined up to a scalar because this corresponds to the conic through the intersection of $Q_{1}^{+}(u, v, w) Q_{3}^{+}(u, v, w)=0$ with $C$. It is then straightforward to find scalars $\lambda, \mu$ such that

$$
\lambda Q_{1}^{+}(u, v, w) Q_{3}^{+}(u, v, w)-\mu Q_{2}^{+}(u, v, w)^{2}
$$

equals the equation for $C$ or, equivalently, that

$$
\lambda Q_{1}^{+}(u, v, w) \mu Q_{3}^{+}(u, v, w)-\left(\mu Q_{2}^{+}(u, v, w)\right)^{2}
$$

defines the curve $C$.

Note that we may find $Q_{i}^{+}$that are quadratic conjugate over the base field we are working with, because of the arbitrary coordinate choice we made when insisting that $G=u^{2} Q(u, v, w)$. The analysis from $\S 3$ guarantees us that by change of basis (corresponding to $\operatorname{Aut}\left(\Gamma^{-}\right)$or, equivalently, fractional linear transformations of $x$ ) we can in fact obtain rational $Q_{1}^{+}, Q_{3}^{+}$. This yields the following amusing result, which is equivalent to saying that all Jacobians of genus 2 curves over $\mathbb{Q}$ occur as Prym varieties of non-hyperelliptic curves of genus 3 over $\mathbb{Q}$.

Proof of Proposition 1.4. Take the curve of genus 2

$$
F: y^{2}=f(x)
$$

and take a sufficiently general plane section of the associated Kummer surface. Using the construction above, we obtain a cover $D \rightarrow C$ such that $\operatorname{Prym}(D / C)=\operatorname{Jac}(F)$. Section 3 tells us that $F$ must be of the described form and the outline above explains how one can find the representation explicitly. 


\section{The ARIthmetic of Prym Varieties in Genus 3}

In fact, the model of $C$ as a plane section of a Kummer surface $\mathcal{K}$ completely encodes the 28 bitangents of $C$ as well. The 16 tropes of $\mathcal{K}$ cut out bitangents on $C$. The remaining 12 bitangents come in pairs, making up the six singular conics in the family $Q_{1}^{+}+2 x Q_{2}^{+}+x^{2} Q_{3}$.

This gives us a way to search for genus 3 curves with all bitangents rational. First, start with a Kummer surface $\mathcal{K}$ with 16 rational tropes (i.e. the Kummer surface of the Jacobian of a genus 2 curve with six rational Weierstraß points). Then, select a plane $V$ such that $C:=V \cap \mathcal{K}$ is of the form

$$
Q_{1}^{+}(u, v, w) Q_{3}^{+}(u, v, w)=Q_{2}^{+}(u, v, w)^{2},
$$

where the singular conics in $Q_{1}^{+}+2 x Q_{2}^{+}+x^{2} Q_{3}$ are split.

Remark 7.1. These conics are all split or non-split simultaneously. In order to see that, we have to look a bit closer to the relation between $\operatorname{Jac}(C)[2]$ and pairs of bitangents. Each of the 63 non-trivial 2 -torsion points can be represented by a pair of bitangents in six ways. Furthermore, the bitangents of $C$ split over the same extension as $\operatorname{Jac}(C)[2]$.

The 16 tropes give rise to at least $\left(\begin{array}{c}16 \\ 2\end{array}\right) / 6=20$ non-trivial rational points in $\operatorname{Jac}(C)[2]$. Thus, $\operatorname{Jac}(C)[2](K) \supset(\mathbb{Z} / 2)^{5}$ and has index at most two in $\operatorname{Jac}(C)[2](\bar{K})$. It follows that the action of Galois on $\operatorname{Jac}(C)[2](\bar{K})$ factors through a group of order at most two. Hence, if not all bitangents are rational over $K$, then they must be conjugate over the one quadratic extension corresponding to this group of order two.

\section{Example}

A curve of genus 3 with all 28 bitangents rational. Take

$$
F: y^{2}=x(x-2)(x-1)(x+1)(x+3) .
$$

The corresponding Kummer surface is

$$
\begin{aligned}
\mathcal{K} & : 36 k_{1}^{4}+84 k_{1}^{3} k_{3}-24 k_{1}^{2} k_{2} k_{3}-12 k_{1}^{2} k_{2} k_{4}+65 k_{1}^{2} k_{3}^{2}+4 k_{1}^{2} k_{3} k_{4}-24 k_{1} k_{2}^{2} k_{3}+4 k_{1} k_{2} k_{3}^{2} \\
& +14 k_{1} k_{2} k_{3} k_{4}+14 k_{1} k_{3}^{3}-4 k_{1} k_{3}^{2} k_{4}-4 k_{1} k_{3} k_{4}^{2}+k_{2}^{2} k_{4}^{2}-2 k_{2} k_{3}^{2} k_{4}+k_{3}^{4}=0 .
\end{aligned}
$$

We take the plane section

$$
V: k_{1}+k_{2}+t k_{3}+k_{4}=0 .
$$

Projecting onto $(u: v: w)=\left(k_{1}: k_{2}: k_{3}\right)$, we obtain

$$
C: Q_{1}^{+}(u, v, w) Q_{3}^{+}(u, v, w)=Q_{2}^{+}(u, v, w)^{2},
$$

where

$$
\begin{aligned}
Q_{1}^{+}= & (36 t-82) u^{2}+(6 t-80) u v+(-11 t+14) u w+(6 t+2) v^{2}+(t+14) v w+t w^{2}, \\
Q_{2}^{+}= & (-3 t+40) u^{2}+\left(-\frac{1}{2} t-9\right) u v+\left(-6 t^{2}+\frac{11}{2} t+51\right) u w+\left(-\frac{1}{2} t-7\right) v^{2} \\
& +\left(-\frac{1}{2} t^{2}-2 t+2\right) v w+\left(-t^{2}+\frac{1}{2} t+7\right) w^{2}, \\
Q_{3}^{+}= & (6 t+2) u^{2}+(t+14) u v+\left(t^{2}+2 t-4\right) u w+t v^{2}+\left(2 t^{2}-t-14\right) v w+\left(t^{3}-t^{2}-8 t+2\right) w^{2} .
\end{aligned}
$$

The 16 bitangents coming from the tropes are given by the polynomials

$$
\begin{aligned}
& u, \quad w, \quad 4 u-2 v+w, \quad 5 u+3 v+(t+1) w, \quad 4 u+v+(t+2) w, \quad u+7 v+(t-1) w, \\
& u-v+w, \quad 7 u+v+(-t-3) w, \quad 9 u+3 v+w, \quad 7 u+v+(t+1) w, \\
& 5 u-v+(-t+1) w, \quad u-v+(-t+3) w, \quad 2 u-4 v+(-t+2) w, \\
& 10 u-2 v+(t-4) w, \quad u+v+w, \quad 2 u+2 v+t w .
\end{aligned}
$$




\section{N. BRUIN}

The remaining 12 bitangents come from the six singular quadrics. They are split if $196+20 t-23 t^{2}$ is a square. Therefore, we substitute

$$
t:=\frac{4 s^{2}-10 s-6}{2 s^{2}+s+3}
$$

and obtain the bitangents given by the polynomials

$$
\begin{aligned}
& (s+21) u+(7 s+3) v+(s-3) w, \quad(10 s+11) u+(-2 s+5) v+(-2 s-1) w, \\
& \left(8 s^{3}+2 s^{2}+11 s-3\right) u+\left(2 s^{3}+5 s^{2}+5 s+6\right) v+\left(8 s^{3}+17 s^{2}-s-6\right) w, \\
& \left(10 s^{3}-s^{2}+12 s-9\right) u+\left(-2 s^{3}-7 s^{2}-6 s-9\right) v+\left(-2 s^{3}+5 s^{2}+30 s-9\right) w, \\
& \left(4 s^{3}+4 s^{2}+7 s+3\right) u+\left(2 s^{2}+s+3\right) v+\left(2 s^{2}+3 s-3\right) w, \\
& \left(2 s^{3}-5 s^{2}-9\right) u+\left(-2 s^{3}-3 s^{2}-4 s-3\right) v+\left(2 s^{3}+7 s^{2}+4 s-1\right) w, \\
& \left(2 s^{3}+7 s^{2}+6 s+9\right) u+\left(2 s^{3}+s^{2}+3 s\right) v+\left(2 s^{3}+s^{2}-3 s\right) w, \\
& \left(4 s^{3}+10 s^{2}+10 s+12\right) u+\left(-2 s^{3}+s^{2}-2 s+3\right) v+\left(s^{3}+4 s^{2}-5 s\right) w, \\
& \left(4 s^{3}+16 s^{2}+13 s+21\right) u+\left(-4 s^{3}-5 s+3\right) v+\left(4 s^{3}+16 s^{2}-3 s-3\right) w, \\
& \left(10 s^{3}+23 s^{2}+24 s+27\right) u+\left(6 s^{3}+s^{2}+8 s-3\right) v+\left(6 s^{3}-5 s^{2}-20 s+7\right) w, \\
& \left(14 s^{3}+13 s^{2}+24 s+9\right) u+\left(2 s^{3}-5 s^{2}-9\right) v+\left(-10 s^{3}-35 s^{2}+9\right) w, \\
& \left(4 s^{3}-8 s^{2}+s-15\right) u+\left(4 s^{3}+4 s^{2}+7 s+3\right) v+\left(4 s^{3}-8 s^{2}-23 s+9\right) w .
\end{aligned}
$$

\section{Applications to finding rational points on curves of genus 3}

In this section, we will apply the concepts of covering collections (see [Bru02, BF05, CW32, Wet97]) and Chabauty methods (see [Col85, Fly97]) to a curve $C$ of genus 3 with an unramified double cover $D$. We end up determining the rational points on a curve of genus 5 inside the Jacobian of a curve $F$ of genus 2. The hardest piece of information we need is the Mordell-Weil group of $\operatorname{Jac}(F)$. Computationally, this is much more attractive than applying Chabauty methods directly to an embedding of $C$ in its own Jacobian. In the latter case, we would have to analyze the Mordell-Weil group of $\operatorname{Jac}(C)$.

In addition, the techniques we present here do not depend on the existence of an embedding of $C$ in $\operatorname{Jac}(C)$. As a result, we see that we can even use the construction to exhibit part of a local-global obstruction for $C$ and $D$ having rational points.

Let $K$ be a number field and let $C$ be a non-hyperelliptic curve of genus 3 with an unramified double cover $D$ over $K$. As we have seen in $\S 3$, it follows that there exists a smooth plane model of $C$ of the form

$$
Q_{1}(u, v, w) Q_{3}(u, v, w)=Q_{2}(u, v, w)^{2},
$$

where $Q_{1}, Q_{2}, Q_{3} \in K[u, v, w]$ are quadratic forms. Without loss of generality, we can assume that $Q_{1}, Q_{2}, Q_{3}$ have integral coefficients. Furthermore, we have a collection of twists of $D$, each covering $C$, of the form

$$
D_{\delta}:\left\{\begin{array}{l}
Q_{1}(u, v, w)=\delta r^{2}, \\
Q_{2}(u, v, w)=\delta r s, \\
Q_{3}(u, v, w)=\delta s^{2} .
\end{array}\right.
$$

We write $\mathcal{O}=\mathcal{O}_{K}$ for the ring of integers of $K$ and we consider the projective $\mathcal{O}_{K}$-scheme $X$ corresponding to the ideal $I=\left(Q_{1}, Q_{3}, Q_{1} Q_{3}-Q_{2}^{2}\right) \mathcal{O}_{K}[u, v, w]$. Since $C$ is non-singular as a curve over $K$, we have that $X \times \mathcal{O}_{K} \operatorname{Spec}(K)$ is empty. Let $S$ be a finite set of primes of $\mathcal{O}$ containing all primes of even residue characteristic such that for any prime $\mathfrak{p}$ of $\mathcal{O}_{K}$ not in $S$, we have that 
$X \times \mathcal{O}_{K} \operatorname{Spec}\left(\mathcal{O}_{K} / \mathfrak{p}\right)$ is empty. Such a set $S$ is easily computed. Let $\operatorname{Res}_{v}(P, Q)$ be the resultant of the polynomials $P, Q$ with respect to the variable $v$. Compute

$$
\operatorname{Res}_{u}\left(\operatorname{Res}_{v}\left(Q_{1}, Q_{3}\right), \operatorname{Res}_{v}\left(Q_{1}, Q_{2}\right)\right)=\lambda w^{16} .
$$

One can take $S$ to be the set of prime divisors of $\lambda$, together with the primes above two. One may obtain a smaller set by intersecting such sets $S$ obtained from all different combinations in which such resultants could be taken. It is straightforward to check that for any point $P \in C(K)$ and any $\mathfrak{p} \notin S$ we have that $\nu_{\mathfrak{p}}\left(Q_{1}(P)\right) \in 2 \mathbb{Z}$ or $\nu_{\mathfrak{p}}\left(Q_{3}(P)\right) \in 2 \mathbb{Z}$, because otherwise $P$ would reduce to a point on $X \times \mathcal{O}_{K} \operatorname{Spec}\left(\mathcal{O}_{K} / \mathfrak{p}\right)$. We recall the definition

$$
K(S, 2):=\left\{\delta \in K^{*}: v_{\mathfrak{p}}(\delta) \equiv 0 \bmod 2 \text { for all primes of } K \text { satisfying } \mathfrak{p} \notin S\right\} / K^{* 2} .
$$

This is a finite subgroup of $K^{*} / K^{* 2}$ and we will identify its elements with a finite set of representatives in $K^{*}$.

We obtain the standard lemma, which determines to the twists $D_{\delta}$ to which rational points of $C$ may lift.

Lemma 8.1. Let $C$ and $Q_{1}, Q_{2}, Q_{3}$ and $S$ be as above. If $\left(u_{0}: v_{0}: w_{0}\right) \in C(K)$, then there exists $\delta \in K(S, 2)$ and $r_{0}, s_{0} \in K$ such that

$$
\left(u_{0}: v_{0}: w_{0}: r_{0}: s_{0}\right) \in D_{\delta}(K) .
$$

Thus, in order to determine the rational points of $C$, it suffices to determine the rational points of $D_{\delta}$ for all $\delta \in K(S, 2)$. From $\S 4$ we know that for

$$
F_{\delta}: y^{2}=-\delta \operatorname{det}\left(Q_{1}+2 x Q_{2}+x^{2} Q_{3}\right),
$$

we have $\operatorname{Prym}\left(D_{\delta} / C\right) \simeq \operatorname{Jac}\left(F_{\delta}\right)$ and Proposition 6.2 gives an explicitly computable map $\varphi: D_{\delta} \rightarrow$ $\operatorname{Jac}\left(F_{\delta}\right)$. We can then proceed to determine $\varphi\left(D_{\delta}(\mathbb{Q})\right) \cap \operatorname{Jac}\left(F_{\delta}\right)(\mathbb{Q})$ or rather, as it turns out, $k\left(\operatorname{Jac}\left(F_{\delta}\right)(\mathbb{Q})\right) \cap k \varphi\left(D_{\delta}\right)(\mathbb{Q})$.

\section{Example}

\section{Chabauty using Prym varieties.}

Proof of Proposition 1.1. See [Bru04] for a transcript of the computer calculations. Applying the method described above we verify that we can take $S=\{1,2,5\}$ and local considerations show that $D_{\delta}(\mathbb{Q})=\emptyset$ for $\delta \neq-1$. We find

$$
F: y^{2}=x^{5}+8 x^{4}-7 x^{3}-\frac{7}{2} x^{2}+5 x-1
$$

and

$$
\operatorname{Jac}(F)(\mathbb{Q})=\langle\mathcal{D}\rangle=\langle[(2 \sqrt{2}-2,17 \sqrt{2}-25)+(-2 \sqrt{2}-2,-17 \sqrt{2}-25)-2 \infty]\rangle .
$$

The equation of the associated Kummer surface is

$$
\begin{aligned}
\mathcal{K} & : 11 k_{1}^{4}-28 k_{1}^{3} k_{2}+70 k_{1}^{3} k_{3}+4 k_{1}^{3} k_{4}+32 k_{1}^{2} k_{2}^{2}-164 k_{1}^{2} k_{2} k_{3}-10 k_{1}^{2} k_{2} k_{4}+171 k_{1}^{2} k_{3}^{2} \\
& +14 k_{1}^{2} k_{3} k_{4}+4 k_{1} k_{2}^{3}-20 k_{1} k_{2}^{2} k_{3}+14 k_{1} k_{2} k_{3}^{2}+14 k_{1} k_{2} k_{3} k_{4}+14 k_{1} k_{3}^{3}-32 k_{1} k_{3}^{2} k_{4} \\
& -4 k_{1} k_{3} k_{4}^{2}+k_{2}^{2} k_{4}^{2}-2 k_{2} k_{3}^{2} k_{4}+k_{3}^{4}=0
\end{aligned}
$$

and, using the interpolation procedure described in $\S 6$, we find that the model of $C$ in $\mathcal{K}$ given by $k \varphi(D)$ is given by the polynomial equation

$$
\begin{aligned}
\psi= & 429136 k_{1}^{4}+1330784 k_{1}^{3} k_{3}+567232 k_{1}^{3} k_{4}-159200 k_{1}^{2} k_{2}^{2}-2866016 k_{1}^{2} k_{2} k_{3}+33440 k_{1}^{2} k_{2} k_{4} \\
& +4248768 k_{1}^{2} k_{3}^{2}+27552 k_{1}^{2} k_{3} k_{4}+881664 k_{1}^{2} k_{4}^{2}+288072 k_{1} k_{2}^{3}-777432 k_{1} k_{2}^{2} k_{3}-256928 k_{1} k_{2}^{2} k_{4} \\
& +244832 k_{1} k_{2} k_{3}^{2}+907424 k_{1} k_{2} k_{3} k_{4}-745472 k_{1} k_{2} k_{4}^{2}+593152 k_{1} k_{3}^{3}-991488 k_{1} k_{3}^{2} k_{4}
\end{aligned}
$$




\section{N. BRUIN}

$$
\begin{aligned}
& +357440 k_{1} k_{3} k_{4}^{2}+573440 k_{1} k_{4}^{3}+34895 k_{2}^{4}-69720 k_{2}^{3} k_{3}+1120 k_{2}^{3} k_{4}+151704 k_{2}^{2} k_{3}^{2} \\
& -364448 k_{2}^{2} k_{3} k_{4}+226032 k_{2}^{2} k_{4}^{2}-251552 k_{2} k_{3}^{3}+569376 k_{2} k_{3}^{2} k_{4}+10752 k_{2} k_{3} k_{4}^{2}-315392 k_{2} k_{4}^{3} \\
& +156704 k_{3}^{4}-167552 k_{3}^{3} k_{4}-283136 k_{3}^{2} k_{4}^{2}+200704 k_{3} k_{4}^{3}+114688 k_{4}^{4}=0 .
\end{aligned}
$$

Using that $\varphi: D \rightarrow \operatorname{Jac}(F)$ is defined over $\mathbb{Q}$ and that $\operatorname{Jac}(F)(\mathbb{Q})=\langle\mathcal{D}\rangle$, we find that a rational point $P \in \varphi(D)(\mathbb{Q})$ must be of the form $P=n \mathcal{D}$ for some $n \in \mathbb{Z}$. Furthermore, considering $F$ and $\mathcal{K}$ over $\mathbb{F}_{13}$, we find that any such point must have $n \equiv \pm 1 \bmod 10$.

Since $\operatorname{Jac}(F)$ is an algebraic group, we can pick a set of local coordinates $z_{1}, z_{2}$ on $\operatorname{Jac}(F)$ around the origin $O$. We can find formal power series vectors $\log \left(z_{1}, z_{2}\right) \in \mathbb{Q}\left[\left[z_{1}, z_{2}\right]\right]^{2}$ and $\operatorname{Exp}\left(l_{1}, l_{2}\right) \in$ $\mathbb{Q}\left[\left[l_{1}, l_{2}\right]\right]^{2}$ such that for any two points $P, Q \in \operatorname{Jac}(F)\left(\mathbb{Q}\left[\left[z_{1}, z_{2}\right]\right]\right)$ we have

$$
\left(z_{1}(P+Q), z_{2}(P+Q)\right)=\operatorname{Exp}\left(\log \left(z_{1}(P), z_{2}(P)\right)+\log \left(z_{1}(Q), z_{2}(Q)\right)\right) .
$$

Furthermore, if $p>2$ is a prime of good reduction then for points in the kernel of reduction

$$
\operatorname{Jac}(F)\left(\mathbb{Q}_{p}\right)^{(1)}=\operatorname{ker}\left(\operatorname{Jac}(F)\left(\mathbb{Q}_{p}\right) \rightarrow \operatorname{Jac}(F)\left(\mathbb{F}_{p}\right)\right),
$$

these power series actually converge and yield an isomorphism

$$
\operatorname{Jac}(F)\left(\mathbb{Q}_{p}\right)^{(1)} \simeq\left(p \mathbb{Z}_{p}\right)^{2} .
$$

Using this, we obtain a power series

$$
\psi(N)=\psi(k((1+10 N) \mathcal{D}))=\psi(k(\mathcal{D}+\operatorname{Exp}(N \log (10 \mathcal{D})))),
$$

say,

$$
\psi(N)=\psi_{0}+\psi_{1} N+\psi_{2} N^{2}+\cdots \in \mathbb{Z}_{13} \llbracket N \rrbracket,
$$

with $\psi_{i} \equiv 0 \bmod 13^{i}$ such that, if $P=(1+10 N) \mathcal{D}$ is a point on $\varphi(D)(K)$, then $\psi(N)=0$.

Note that the values of $\psi(k(\mathcal{D})), \psi(k(11 \mathcal{D}))$ determine $\psi_{0}, \psi_{1} \bmod 13^{2}$, so one does not need an explicit description of the formal group law on $\operatorname{Jac}(F)$ to obtain an approximation to $\psi(N)$.

Since $\psi(k(\mathcal{D}))=0$ and $\psi(k(11 \mathcal{D})) \not \equiv 0 \bmod 13^{2}$, it follows that $\psi_{1} \not \equiv 0 \bmod 13^{2}$. From Straßmann's lemma it follows that $\psi(N)$ has at most one zero for $N \in \mathbb{Z}_{13}$ (i.e. $N=0$ ). This implies that $\varphi(D)$ has only one rational point which reduces to $\mathcal{D}$ modulo 13. By symmetry, it follows that there is also only one rational point reducing to $-\mathcal{D}$ modulo 13 . On the other hand, the computation over $\mathbb{F}_{13}$ shows that all rational points of $\varphi(D)$ reduce to $\pm \mathcal{D}$ modulo 13 . Hence, it follows that

$$
\varphi(D)(K)=\{\mathcal{D},-\mathcal{D}\}
$$

and that $C$ has only one rational point, being $(0: 1: 0)$.

\section{Example}

Computations in the Brauer-Manin obstruction. Since the embedding of $D$ in $\operatorname{Prym}(D / C)$ is independent of $D$ having any rational points, we can also apply this construction to curves $D$ that have no rational points, but do have rational points everywhere locally. Using information obtained from the reduction of $\operatorname{Jac}(F)$ at various primes, we might actually succeed in proving that $D(\mathbb{Q})$ is empty. Under the assumption that $\operatorname{Jac}(D)$ has a finite Tate-Shafarevich group, this corresponds to computing part of the Brauer-Manin obstruction according to [Sch99].

We consider the curve

$$
C:\left(v^{2}+v w-w^{2}\right)\left(u v+w^{2}\right)=\left(u^{2}-v^{2}-w^{2}\right)^{2} .
$$

It is easily checked that $C$ has points everywhere locally. Furthermore, the set of primes $S$ described above can be taken to be $\{2\}$ and $D_{\delta}$ only has points everywhere locally for $\delta=1$. 
Proof of Proposition 1.3. See [Bru04] for a transcript of the computer calculations. The fact that $D\left(\mathbb{Q}_{p}\right)$ and $D(\mathbb{R})$ are all non-empty can be verified with a straightforward computation. To prove that $D(\mathbb{Q})=\emptyset$ we map $D$ into $\operatorname{Prym}(D / C)=\operatorname{Jac}(F)$, where

$$
F: y^{2}=x^{6}+2 x^{5}+15 x^{4}+40 x^{3}-10 x .
$$

We write $\infty^{+}$and $\infty^{-}$for the two points above $x=\infty$ on the desingularization of $F$. We find that

$$
\operatorname{Jac}(F)(\mathbb{Q})=\left\langle\mathcal{D}_{1}, \mathcal{D}_{2}\right\rangle \simeq \mathbb{Z} \times \mathbb{Z},
$$

where

$$
\begin{aligned}
& \mathcal{D}_{1}=\left[\infty^{+}-\infty^{-}\right] \\
& \mathcal{D}_{2}=\left[\left\{x^{2}+\frac{2}{5} x+\frac{1}{41}=0, y=\frac{4683}{2050} x-\frac{281}{410}\right\} \cdot F-\kappa_{F}\right] .
\end{aligned}
$$

Considering $\operatorname{Jac}(F)$ modulo 7 , we find

$$
\varphi(D)(\mathbb{Q}) \subset\left\{ \pm 9 \mathcal{D}_{1}, \pm 22 \mathcal{D}_{1}, \pm 23 \mathcal{D}_{1}\right\}+\left\langle 55 \mathcal{D}_{1}, \mathcal{D}_{2}-15 \mathcal{D}_{1}\right\rangle
$$

and considering $\operatorname{Jac}(F)$ modulo 11, we find

$$
\varphi(D)(\mathbb{Q}) \subset\left\{ \pm 33 \mathcal{D}_{1}\right\}+\left\langle 93 \mathcal{D}_{1}, \mathcal{D}_{2}+46 \mathcal{D}_{1}\right\rangle .
$$

Considering $\operatorname{Jac}(F)$ modulo $11^{2}$, we find that the two residue classes modulo 11 lift to 11 residue class modulo

$$
\left\langle 11 \cdot 93 \mathcal{D}_{1}, 11 \cdot\left(\mathcal{D}_{2}+46 \mathcal{D}_{1}\right)\right\rangle .
$$

We combine the information modulo $11^{2}$ and 7 and express it as congruences modulo

$$
\left\langle 55 \mathcal{D}_{1}, \mathcal{D}_{2}-15 \mathcal{D}_{1}, 11 \cdot 93 \mathcal{D}_{1}, 11 \cdot\left(\mathcal{D}_{2}+46 \mathcal{D}_{1}\right)\right\rangle=\left\langle 11 \mathcal{D}_{1}, \mathcal{D}_{2}-4 \mathcal{D}_{1}\right\rangle
$$

We obtain

$$
\begin{array}{llll}
\text { from } 7 & : \varphi(D)(\mathbb{Q}) & \subset\left\{0, \pm \mathcal{D}_{1}, \pm 2 \mathcal{D}_{1}\right\} & +\left\langle 11 \mathcal{D}_{1}, \mathcal{D}_{2}-4 \mathcal{D}_{1}\right\rangle \\
\text { from } 11^{2} & : \varphi(D)(\mathbb{Q}) & \subset\left\{ \pm 4 \mathcal{D}_{1}\right\} & +\left\langle 11 \mathcal{D}_{1}, \mathcal{D}_{2}-4 \mathcal{D}_{1}\right\rangle
\end{array}
$$

It follows that $D(\mathbb{Q})=\emptyset$ and therefore that $C(\mathbb{Q})$ is empty as well.

\section{ACKNOWLEDGEMENTS}

I would like to thank MSRI for their 2000 Fall special semester on explicit methods in number theory for bringing me into contact with many mathematicians. I have greatly benefited from the discussions I had with them. In particular, I would like to thank, Armand Brumer, J. Merriman, Ed Schaefer, Michael Stoll and Joe Wetherell. Furthermore, I would like to thank the anonymous referee for many constructive comments.

\section{REFERENCES}

ACGH85 E. Arbarello, M. Cornalba, P. A. Griffiths and J. Harris, Geometry of algebraic curves, Vol. I, Grundlehren der Mathematischen Wissenschaften, vol. 267 (Springer, New York, 1985).

BCP97 W. Bosma, J. Cannon and C. Playoust, The Magma algebra system. I. The user language, in Computational algebra and number theory, London, 1993, J. Symbolic Comput. 24 (1997), 235-265.

Bru02 N. R. Bruin, Chabauty methods and covering techniques applied to generalized Fermat equations, of CWI Tract, vol. 133 (Stichting Mathematisch Centrum Centrum voor Wiskunde en Informatica, Amsterdam, 2002).

Bru03 N. Bruin, Chabauty methods using elliptic curves, J. reine angew. Math. 562 (2003), 27-49. 


\section{The ARIthmetic of Prym VARIETIEs in Genus 3}

Bru04 N. Bruin, Routines and transcript of computations, http://www.cecm.sfu.ca/ ${ }^{\text {bruin/g3prym }}$ (2004).

BF05 N. Bruin and E. V. Flynn, Towers of 2-covers of hyperelliptic curves, Trans. Amer. Math. Soc. 357 (2005), 4329-4347 (electronic).

BF03 N. Bruin and E. V. Flynn, n-covers of hyperelliptic curves, Math. Proc. Cambridge Philos. Soc. 134 (2003), 397-405.

CF96 J. W. S. Cassels and E. V. Flynn, Prolegomena to a Middlebrow arithmetic of curves of genus 2, London Mathematical Society, Lecture Notes Series, vol. 230 (Cambridge University Press, Cambridge, 1996).

Cay79 A Cayley, On the bitangents of a quartic, in A treatise on the higher plane curves (Chelsea, New York, 1879), 387-389.

CW32 C. Chevalley and A. Weil, Un théorème d'arithmétiques sur les courbes algébriques, C. R. Math. Acad. Sci. Paris 195 (1932), 570-572.

Col85 R. F. Coleman, Effective Chabauty, Duke Math. J. 52 (1985), 765-770.

Don92 R. Donagi, The fibers of the Prym map, in Curves, Jacobians, and abelian varieties, Amherst, MA, 1990, Contemporary Mathematics, vol. 136 (American Mathematical Society, Providence, RI, 1992), 55-125.

Edg94 W. L. Edge, 28 real bitangents, Proc. Roy. Soc. Edinburgh Sect. A 124 (1994), 729-736.

Fly90 E. V. Flynn, The jacobian and formal group of a curve of genus 2 over an arbitrary ground field, Math. Proc. Cambridge Philos. Soc. 107 (1990), 425-441.

Fly96 E. V. Flynn, Electronic resources for curves of genus 2, ftp://ftp.liv.ac.uk/pub/genus2/ (1996).

Fly97 E. V. Flynn, A flexible method for applying Chabauty's theorem, Compositio Math. 105 (1997), 79-94.

Ful69 W. Fulton, Algebraic curves. An introduction to algebraic geometry, Notes written with the collaboration of Richard Weiss, Mathematics Lecture Notes Series (Benjamin, New York, 1969).

Har77 R. Hartshorne, Algebraic geometry, Graduate Texts in Mathematics, vol. 52 (Springer, New York, 1977).

Mum74 D. Mumford, Prym varieties. I, in Contributions to analysis (a collection of papers dedicated to Lipman Bers) (Academic Press, New York, 1974), 325-350.

Rec74 S. Recillas, Jacobians of curves with $g_{4}^{1}$ 's are the Prym's of trigonal curves, Bol. Soc. Mat. Mexicana (2) 19 (1974), 9-13.

Sch98 E. F. Schaefer, Computing a Selmer group of a Jacobian using functions on the curve, Math. Ann. 310 (1998), 447-471.

Sch99 V. Scharaschkin, Local-global problems and the Brauer-Manin obstruction, PhD thesis, University of Michigan (1999).

Sko01 A. Skorobogatov, Torsors and rational points, Cambridge Tracts in Mathematics (Cambridge University Press, Cambridge, 2001).

Ver87 A. Verra, The fibre of the Prym map in genus three, Math. Ann. 276 (1987), 433-448.

Wet97 J. L. Wetherell, Bounding the number of rational points on certain curves of high rank, $\mathrm{PhD}$ thesis, U. C. Berkeley, 1997.

Nils Bruin bruin@member.ams.org

Department of Mathematics, Simon Fraser University, Burnaby, BC, Canada V5A 1S6 